\title{
Two-Timeslot Two-Way Full-Duplex Relaying for 5G Wireless Communication Networks
}

\author{
Zhengquan Zhang, Zheng Ma, Member, IEEE, Ming Xiao, Senior Member, IEEE, George \\ K. Karagiannidis, Fellow, IEEE, Zhiguo Ding, Senior Member, IEEE, and Pingzhi Fan, Fellow, IEEE
}

\begin{abstract}
We propose a novel two-timeslot two-way full-duplex (FD) relaying scheme, in which the access link and the backhaul link are divided in the time domain, and we study the average end-to-end rate and the outage performance. According to the user equipment (UE) capability and services, we investigate two scenarios: three-node $I$ - and four-node $Y$-relaying channels. Among various relaying protocols, the well-known amplify-andforward (AF) and decode-and-forward (DF), are considered. Closed-form expressions for the average end-to-end rate and the outage probability, under the effect of residual self-interference and inter-user interference, are presented. The results show that the proposed two-timeslot two-way FD relaying scheme can achieve higher rate and better outage performance than the half-duplex one, when residual self-interference is below a certain level. Therefore, the two-timeslot two-way FD relaying scheme can achieve reasonable tradeoff between performance and complexity, and so, it will be an efficient solution for the fifth-generation $(5 \mathrm{G})$ wireless communication systems.
\end{abstract}

Index Terms-Full-duplex, two-way relaying, time division multiplexing, residual self-interference, $Y$-relaying channel.

\section{INTRODUCTION}

$\mathbf{R}$ ELAYING technique, which enables the wireless networks to work in a cooperative manner, is an efficient way to improve spectrum efficiency and extend coverage. Halfduplex (HD) relaying has already been adopted as one of the key features in long term evolution-advanced (LTE-A) systems [1]. Field tests have already been performed and the results show that relays improve the coverage in the holes and the data rate in the poorly covered area of a donor eNodeB (DeNB) [2], [3]. To satisfy the increasing data rate demands, the research on the fifth generation $(5 \mathrm{G})$ wireless communication systems is on-going both in academic and industry. In the $5 \mathrm{G}$ era, it will

This work was supported by the National Basic Research Program of China (No. 2012CB316100), National Natural Science Foundation of China (No. 61571373), National High-tech R\&D Program of China (No.2014AA01A707), Key Grant Project of Chinese Ministry of Education (No.311031100), and Young Innovative Research Team of Sichuan Province (No. 2011JTD0007).

Z. Zhang, Z. Ma and P. Fan are with the Key Lab of Information Coding and Transmission, Southwest Jiaotong University, Chengdu, 610031, China (e-mail: zhang.zhengquan@ @otmail.com; zma@ home.swjtu.edu.cn; pzfan@home.swjtu.edu.cn).

M. Xiao is with the Communication Theory Lab, School of Electrical Engineering and the ACCESS Linnaeus Center, Royal Institute of Technology, Sweden (e-mail: mingx@kth.se).

G. K. Karagiannidis is with the Key Lab of Information Coding and Transmission, Southwest Jiaotong University, Chengdu, 610031, China, and also with the Department of Electrical and Computer Engineering, Aristotle University of Thessaloniki, 54 124, Thessaloniki, Greece (e-mail: geokarag@auth.gr).

Z. Ding is with the School of Computing and Communications, Lancaster University, LA1 4YW, U.K (e-mail: z.ding@lancaster.ac.uk). be required that wireless systems should offer up to $10 \mathrm{Gbps}$ data rate to support a variety of emerging services [4], [5], which stimulates researchers to constantly explore innovative techniques with higher spectrum efficiency. Recently, fullduplex (FD) radio was proposed as a promising technique for the $5 \mathrm{G}$ systems, because it can double the spectrum efficiency by achieving simultaneous transmission and reception on the same carrier frequency [6]-[9]. In order to adapt to the 5G requirements, relaying schemes with high spectrum efficiency, such as two-way, and full-duplex relaying, etc., have been recently attracted considerable attention.

\section{A. Related Work}

Fig. 1 summarizes existing three-node relay transmission schemes, which differ in the number of time slots required to achieve the bidirectional data exchange.

- Four-timeslot HD relaying [10], [11]. As shown in Fig. 1(a), this scheme needs four time slots to achieve the bidirectional data transmission between the user equipment (UE) and the donor base station (BS) via the relay. In the first time slot, the UE transmits uplink data to the relay, while the relay forwards the received data to the BS in the second time slot. Similarly, in the third time slot, the BS transmits downlink data to the relay, while the relay forwards the received data to the UE in the fourth time slot. Among all the relay transmission schemes, this scheme has the lowest complexity, but its spectrum efficiency is the lowest.

- Three-timeslot HD relaying [12], [13]. Fig. 1(b) shows that the bidirectional data transmission between the UE and the BS via the relay, is achieved in three time slots. In the first time slot, the UE transmits uplink data to the relay, while the BS transmits downlink data to the relay in the second time slot. In the third time slot, the relay broadcasts a combination of the received data to the UE and the BS. Generally, network coding [14], [15] is used to achieve the signal combining of the received two data flows at the relay. Therefore, the destination node receives not only the desired data from the source node but also its own previous transmitted data. The destination node subtracts back-propagating interference $(\mathrm{BI})^{1}[16]$ prior to decoding.

\footnotetext{
${ }^{1}$ Back-propagating interference refers to the phenomenon, that signals previously transmitted by a node are propagated back to its receiver via intermediate nodes.
} 
- Two-timeslot HD relaying [16]-[19]. The two time slots bidirectional data transmission, between the UE and the BS via the relay, is demonstrated in Fig. 1(c). This scheme consists of the multiple access (MAC) and broadcast (BC) stages. At the MAC stage, the UE and the BS simultaneously transmit their own data to the relay on the same carrier frequency, while the relay broadcasts a combination of the received data to the UE and the BS at the BC stage. Similarly, both the UE and the BS need to suppress the back-propagating interference in two-timeslot HD relaying.

- One-way FD relaying (Protocol 1) [20]-[24]. In this scheme, unidirectional data transmission between the UE and the BS via the FD relay is achieved in one time slot (See Fig. 1(d)). In order to exchange the bidirectional data between the UE and the BS, it still needs two time slots. In the first time slot, the UE transmits uplink to the BS via the relay, while the BS transmits downlink data to the UE via the relay in the second time slot. In this scheme, only the relay operates in FD mode, and suffers from residual self-interference ${ }^{2}$ [20]. When the amplify-and-forward (AF) protocol is adopted, it is also called as full-duplex repeater [25], [26], which has widely been used in cellular networks. Unlike the BS with whole baseband and radio remote units, a full-duplex repeater consists of a radio receiver and a transmitter, which can enable the signal to cover longer distances without degradation, through retransmitting the received weak signal at a higher power.

- FD relaying (Protocol 2) [27]. As shown in Fig. 1(e), two time slots are used to achieve the bidirectional data exchange between the UE and the BS via the relay. In the first time slot, the UE transmits the uplink data to the relay. In the second time slot, the relay receives the downlink data from the BS, then broadcasts a combination of the received data to the UE and the BS. In this scheme, the relay and the BS operate in FD mode, thus they suffer from residual self-interference generated by the co-channel transmission and imperfect interference cancellation. In addition, both the UE and the BS need to cancel the back-propagating interference as well.

- Two-way FD relaying (Protocol 3) [27]-[29]. Fig. 1(f) demonstrates the bidirectional data exchange between the UE and the BS via the relay in one time slot. The relay simultaneously receives two data flows from the UE and the BS on the same carrier frequency, then broadcasts their combination to the UE and the BS. Compared with the above relaying schemes, two-way FD relaying (Protocol 3) can achieve highest spectrum efficiency, but it also presents the maximum processing complexity. This is because the UE, the relay, and the BS need to suppress residual self-interference created by the co-channel transmission and imperfect interference cancellation, and the back-propagating interference needs

\footnotetext{
${ }^{2}$ Self-interference refers to the phenomenon, that co-channel signals transmitted by a full-duplex node are looped back to its receiver simultaneously. Due to imperfect interference cancellation, loop signals still remains in the receiver and are considered as interference, when decoding the desired data.
}

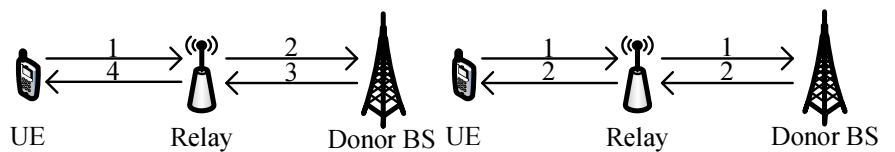

(a) 4-time slot HD relaying

(d) One-way FD relaying (Protocol 1)

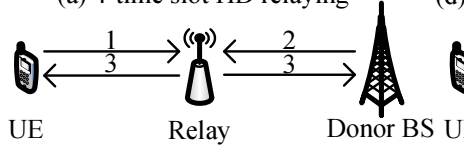

(b) 3-time slot HD relaying

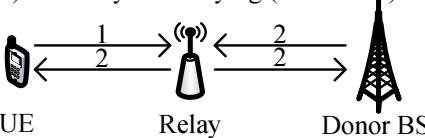

(e) FD relaying (Protocol 2)
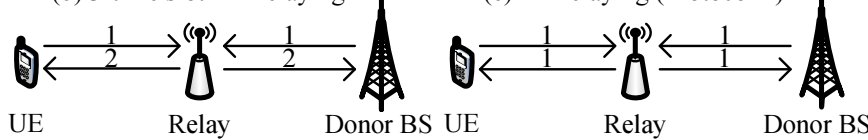

(c) 2-time slot HD relaying

(f) Two-way FD relaying (Protocol 3)

Fig. 1. Summary of existing relay transmission schemes. (a) 4-timeslot HD relaying. (b) 3-timeslot HD relaying. (c) 2-timeslot HD relaying. (d) Oneway FD relaying (Protocol 1); (e) FD relaying (Protocol 2). (f) Two-way FD relaying (Protocol 3)

canceling at both the UE and the BS.

\section{B. Motivation and Contribution}

Even though there are several available relaying schemes described above, it seems that they are not optimal for the future 5G networks. For one-way FD relaying (Protocol 1), the BS operates in HD mode, and thus, does not fully take the advantages of FD; FD relaying (Protocol 2) requires that relay performs signal combining and the UE perfectly cancel the back-propagating interference; the relay needs two sets of FD transceivers to achieve simultaneous bidirectional data exchange between the UE and the BS in two-way FD relaying (Protocol 3) and also performs signal combining. Besides, in this scheme, both the UE and the BS are required to perfectly cancel the back-propagating interference. In addition, the BS cannot dynamically adjust the time resources to optimize the performance for some scenarios, such as the SNR imbalance between the access link and the backhaul link [30].

Motivated by the above, in this paper, we propose and investigate a novel two-timeslot two-way FD relaying scheme based on time division multiplexing, in which the access link and the backhaul link are divided in time domain, and FD operation is introduced in each hop. The available time slots for each hop depend on the configuration from higher layer. In the available time slots for the access link, bidirectional data exchange between the UE and the relay is realized, while the BS can also use these slots to serve users in the BS macro cell. Bidirectional data transmission between the relay and the BS is achieved in the available time slots for the backhaul link. Compared with FD relaying (Protocol 2) and two-way FD relaying (Protocol 3), there is no back-propagating interference in two-timeslot two-way FD relaying scheme.

According to the UE capability and services, two scenarios are considered: a) $I$-relaying channel (IRC); and b) $Y$-relaying channel (YRC) [31]-[33]. In $I$-relaying channel, the UE, the relay, and the base station support FD operation and the UE has concurrent uplink and downlink data, while in $Y$ relaying channel, the BS and the relay, both equipped with FD transceiver, serve the UE1 with uplink data and the UE2 
with downlink data. Among various relaying protocols, we consider the well-known $\mathrm{AF}$ and decode-and-forward (DF) $[16]^{3}$. In contrast to [34], we assume that there is no direct link between the UE and the BS, due to transmit power limitation or the severe shadowing effect [18], [20]. Furthermore, we consider the effect of residual self-interference and inter-user interference [35].

The contribution of this paper can be summarized as follows:

- A novel two-timeslot two-way FD relaying scheme, which divides the access link and the backhaul link in time domain, is proposed, and the scenarios of three-node $I$ - and four-node $Y$-relaying channels are studied;

- Closed-form expressions for the average end-to-end rate and the outage probability of the AF and DF based twotimeslot two-way FD relaying schemes, are derived;

- Detailed analysis and performance comparisons between the proposed scheme and the HD relaying, are also presented.

\section{Paper Outline}

The rest of the paper is organized as follows. Section II describes the system model and explains the main concept of the two-timeslot two-way FD relaying scheme. A detailed derivation of the average end-to-end rate and the outage probability of this relaying scheme for the $I$ - and $Y$-relaying channels, are presented in Section III. Analytical results, Monte Carlo simulations and discussion are presented in Section IV, followed by the conclusions in Section V.

\section{SySTEM MOdEL}

In this paper, a two-timeslot two-way FD relaying model is considered as in Fig. 2, with (a) $I$-relaying channel and (b) $Y$ relaying channel, where the hop between the UE and the relay is referred to as the access link, while the backhaul link refers to the hop between the relay and donor BS. Furthermore, no direct link between source and destination nodes is assumed, due to transmit power limitation or the severe shadowing effect [18], [20]. Also, we consider a simple 1:1 access/backhaul link time slot configuration, where the odd time slots are configured to the access link, while the backhaul link uses the even time slots. That is, in the odd time slots, the simultaneous uplink and downlink data transmission between the UE and the relay on the same carrier frequency, are achieved, while the data exchange between the relay and the BS is implemented in the even time slots.

In the (a) scenario above, all the nodes operate in FD mode, thus they suffer from residual self-interference (SI), because of the co-channel transmission and imperfect interference cancellation. In (b), the relay and the BS operate in FD mode, while the UE1 with uplink data and the UE2 with downlink data

\footnotetext{
${ }^{3}$ The compute-and-forward (CF) protocol enables relays to decode linear equations of the transmitted messages using the noisy linear combinations provided by the channel, which relies on codes with a linear structure, especially nested lattice codes [15], [18]. Thus, CF protocol is based on a different concept than $\mathrm{AF}$ and $\mathrm{DF}$ protocols. We focus on $\mathrm{DF}$ and $\mathrm{AF}$ here for their practical simplicity.
}

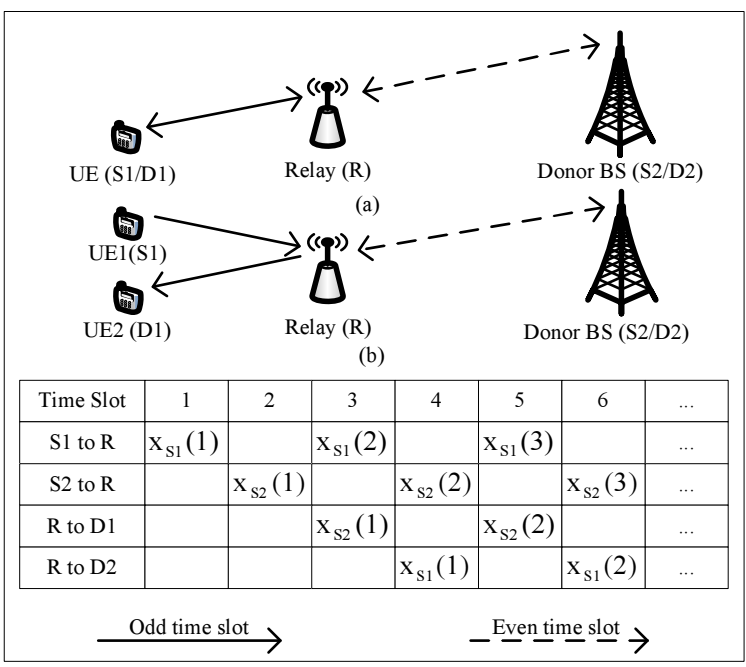

Fig. 2. System model

work in HD mode, thereby inter-user interference between two UEs replaces self-interference generated at the UE in scenario (a). In (a), the UE1 as source, the UE1 as destination, the relay, the donor BS as source, and the donor BS as destination are denoted as $S 1, D 1, R, S 2$, and $D 2$, respectively, while in (b), the UE1, the UE2, the relay, the donor BS as source, and the donor BS as destination are represented by $S 1, D 1, R, S 2$, and $D 2$, respectively.

Although the detrimental effect of self-interference can be mitigated by using multiple-stage interference cancellation [36], there is residual self-interference due to the imperfections of the radio frequency chains. According to [7], [20], [37], the variance of residual self-interference is approximately proportional to the $\lambda$-th power of the average transmitted power, where, $\lambda \in[0,1]$, depends on the effect of the adopted selfinterference cancellation techniques. In practice, the accurate relation between the transmitted power and residual selfinterference is still unknown [37]. Usually, it is set to empirical values, which are obtained from field measurements and can be found in [7].

The involved channels are $\mathrm{S} 1 \rightarrow \mathrm{R}, \mathrm{R} \rightarrow \mathrm{D} 2, \mathrm{~S} 2 \rightarrow \mathrm{R}, \mathrm{R} \rightarrow \mathrm{D} 1$, $\mathrm{S} 1 \rightarrow \mathrm{D} 1, \mathrm{R} \rightarrow \mathrm{R}$, and $\mathrm{S} 2 \rightarrow \mathrm{D} 2$, whose channel coefficients are denoted as $h_{S 1 R}, h_{R D 2}, h_{S 2 R}, h_{R D 1}, h_{S 1 D 1}, h_{R R}$, and $h_{S 2 D 2}$, respectively. Note, that in scenario (a), S1 $\rightarrow \mathrm{D} 1$ and $\mathrm{S} 2 \rightarrow \mathrm{D} 2$ are the residual self-interference channels, while $\mathrm{S} 1 \rightarrow \mathrm{D} 1$ is the inter-user interference channel and $\mathrm{S} 2 \rightarrow \mathrm{D} 2$ is the residual self-interference channel in scenario (b). We consider that $h_{S 1 R}$ and $h_{S 2 R}$ are independent and the channels are reciprocal, thus it holds that $h_{S 1 R}=h_{R D 1}$ and $h_{S 2 R}=h_{R D 2}$ for the $I$-relaying channel, while $h_{S 2 R}=h_{R D 2}$ holds for the $Y$-relaying channel. Similarly to [20], [38], the residual self-interference channels are assumed to be free of fading, while the channels $\mathrm{S} 1 \rightarrow \mathrm{R}, \mathrm{R} \rightarrow \mathrm{D} 2, \mathrm{~S} 2 \rightarrow \mathrm{R}, \mathrm{R} \rightarrow \mathrm{D} 1$, and the inter-user interference (IUI) channel $\mathrm{S} 1 \rightarrow \mathrm{D} 1$ are subjected to Rayleigh fading. Thus, the instantaneous signal-to-noise ratio (SNR), $\gamma$, is an exponential random variable (RV), with 
probability distribution function (PDF) given by [20]

$$
f_{\bar{\gamma}}(\gamma)=\frac{1}{\bar{\gamma}} e^{-\gamma / \bar{\gamma}}
$$

where $\bar{\gamma}$ is the average SNR. The instantaneous channel SNR is, $\gamma=|h|^{2} P / \sigma^{2}$, where $h$ is the channel coefficient and $\sigma^{2}$ is the noise power, while the average channel SNR is, $\bar{\gamma}=\varepsilon\left\{|h|^{2}\right\} P / \sigma^{2}$, where $\varepsilon\{\cdot\}$ denotes expectation [20]. The normalized transmitted powers of the source UE, the relay, and the donor BS are, $P_{1}=1, P_{R}=1$, and $P_{2}=1$, respectively. Also, the instantaneous SNRs of the involved channels are denoted as $\gamma_{S 1 R}, \gamma_{R D 2}, \gamma_{S 2 R}, \gamma_{R D 1}, \gamma_{S 1 D 1}, \gamma_{R R}$, and $\gamma_{S 2 D 2}$, while their corresponding average SNRs are represented by $\bar{\gamma}_{S 1 R}, \bar{\gamma}_{R D 2}, \bar{\gamma}_{S 2 R}, \bar{\gamma}_{R D 1}, \bar{\gamma}_{S 1 D 1}, \bar{\gamma}_{R R}$, and $\bar{\gamma}_{S 2 D 2}$.

\section{Performance Analysis}

\section{A. I-Relaying Channel}

In the odd time slots, $k=2 n-1, n=1,2,3, \ldots$, simultaneous bidirectional data transmission and reception is achieved between the UE1 and the relay. In addition, since both the UE1 and the relay operate in FD mode, they suffer from residual self-interference, because of imperfect interference cancellation. Therefore, the signals received at the relay and the UE1 can be respectively expressed as

$$
y_{R}[k]=h_{S 1 R} x_{S 1}[k]+v_{R}[k]+n_{R}[k],
$$

and

$$
y_{D 1}[k]=h_{R D 1} t_{R}[k]+v_{D 1}[k]+n_{D 1}[k],
$$

where $x_{S_{i}} \sim C N\left(0, P_{i}\right)$ is the transmit symbol of the source node $i=1,2, v_{R} \sim C N\left(0,\left|h_{R R}\right|^{2} P_{R}\right)$ residual selfinterference at the relay, $n_{R} \sim C N\left(0, \sigma_{R}^{2}\right)$ the additive white Gaussian noise (AWGN) at the relay, $t_{R}$ the relay's transmit signal, $v_{D_{i}} \sim C N\left(0,\left|h_{S_{i} D_{i}}\right|^{2} P_{i}\right)$ residual self-interference at the destination node $i=1,2$, and $n_{D_{i}} \sim C N\left(0, \sigma_{D_{i}}^{2}\right)$ the AWGN at the destination node. Note, that $t_{R}$ depends on the relaying scheme, and is given explicitly in the following pages.

Similarly, in the even time slots, $k=2 n, n=1,2,3, \ldots$, simultaneous bidirectional data exchange is achieved between the relay and the donor BS. Both the relay and the donor BS suffer from residual self-interference, because of FD operation and imperfect interference cancellation. So, the signals received at the relay and donor BS can be respectively written as

$$
y_{R}^{\prime}[k]=h_{S 2 R} x_{S 2}[k]+v_{R}[k]+n_{R}[k],
$$

and

$$
y_{D 2}[k]=h_{R D 2} t_{R}[k]+v_{D 2}[k]+n_{D 2}[k] .
$$

1) Amplify-and-Forward: In the odd time slots, the relay receives and buffers uplink data from the UE1, and forwards the buffered downlink data to the UE1 by amplifying the signals, based on the channel gains. Similarly, in the even time slots, the relay receives and buffers downlink data from the donor BS, and forwards the buffered uplink data to the donor BS, with the same processing as with the downlink data forwarding.
In the odd time slots, $k=2 n-1, n=1,2,3, \ldots$, the relay amplifies the input signal received in the even time slots by an amplification factor $\beta>0$, which induces a delay of $\tau$ assumed to be one without loss of generality. Thus, the transmit signal of the relay can be expressed as [20]

$$
\begin{aligned}
t_{R}[k] & =\beta y_{R}[k-\tau] \\
& =\beta\left(h_{S 2 R} x_{S 2}[k-\tau]+v_{R}[k-\tau]+n_{R}[k-\tau]\right) .
\end{aligned}
$$

Considering the average transmit power of the relay, $\varepsilon\left\{\left|t_{R}[k]\right|^{2}\right\}=P_{R}=1$ [29], the amplification factor $\beta$ can be written as [20], [21]

$$
\beta=\left(\left|h_{S 2 R}\right|^{2}+\left|h_{R R}\right|^{2}+\sigma_{R}^{2}\right)^{-1 / 2} .
$$

By substituting (6) into (3), $y_{D 1}[k]$ can be expressed as

$$
\begin{aligned}
y_{D 1}[k]= & h_{R D 1}\left(\beta y_{R}[k-\tau]\right)+v_{D 1}[k]+n_{D 1}[k] \\
= & \beta h_{R D 1}\left(h_{S 2 R} x_{S 2}[k-\tau]+v_{R}[k-\tau]\right. \\
& \left.+n_{R}[k-\tau]\right)+v_{D 1}[k]+n_{D 1}[k] .
\end{aligned}
$$

Therefore, the instantaneous SNR of the signal received at the UE1, can be expressed as

$$
\gamma_{I R C, D 1}^{A F}=\frac{\beta^{2}\left|h_{R D 1}\right|^{2}\left|h_{S 2 R}\right|^{2}}{\beta^{2}\left|h_{R D 1}\right|^{2}\left(\left|h_{R R}\right|^{2}+\sigma_{R}^{2}\right)+\left|h_{S 1 D 1}\right|^{2}+\sigma_{D 1}^{2}} .
$$

Finally, by substituting (7) into (9)

$\gamma_{I R C, D 1}^{A F}=\frac{\gamma_{R D 1} \gamma_{S 2 R}}{\gamma_{R D 1}\left(\bar{\gamma}_{R R}+1\right)+\left(\bar{\gamma}_{S 1 D 1}+1\right)\left(\gamma_{S 2 R}+\bar{\gamma}_{R R}+1\right)}$.

Similarly, in the even time slots, $k=2 n, n=1,2,3, \ldots$, the instantaneous SNR of the signal received at the donor BS is

$\gamma_{I R C, D 2}^{A F}=\frac{\gamma_{R D 2} \gamma_{S 1 R}}{\gamma_{R D 2}\left(\bar{\gamma}_{R R}+1\right)+\left(\bar{\gamma}_{S 2 D 2}+1\right)\left(\gamma_{S 1 R}+\bar{\gamma}_{R R}+1\right)}$.

Compared with HD relaying, the denominator of (10) contains the terms of $\bar{\gamma}_{S 1 D 1}$ and $\bar{\gamma}_{R R}$, while the denominator of (11) contains the terms of $\bar{\gamma}_{S 2 D 2}$ and $\bar{\gamma}_{R R}$. This means that the two-timeslot two-way FD relaying deteriorates the instantaneous SNRs of the end-to-end link, due to residual self-interference at all nodes.

In the AF based two-timeslot two-way FD I-relaying, the average end-to-end rate is defined as

$$
\begin{aligned}
\bar{R}_{I R C}^{A F} & =\frac{\varepsilon\left\{\log _{2}\left(1+\gamma_{I R C, D 1}^{A F}\right)+\log _{2}\left(1+\gamma_{I R C, D 2}^{A F}\right)\right\}}{2} \\
& =\frac{\varepsilon\left\{\log _{2}\left(1+\gamma_{I R C, D 1}^{A F}\right)\right\}+\varepsilon\left\{\log _{2}\left(1+\gamma_{I R C, D 2}^{A F}\right)\right\}}{2} .
\end{aligned}
$$

Due to no spectral loss in FD operation, the pre-log factor is equal to one, which is different from the average end-toend rate for HD relaying. Considering channel reciprocity and identical self-interference assumption, $\bar{R}_{I R C}^{A F}$ can be further written as

$$
\bar{R}_{I R C}^{A F}=\varepsilon\left\{\log _{2}\left(1+\gamma_{I R C, D 1}^{A F}\right)\right\}=\varepsilon\left\{\log _{2}\left(1+\gamma_{I R C, D 2}^{A F}\right)\right\} .
$$

Theorem 1. The average end-to-end rate for the AF based two-timeslot two-way FD I-relaying over Rayleigh fading 
channels, can be expressed as

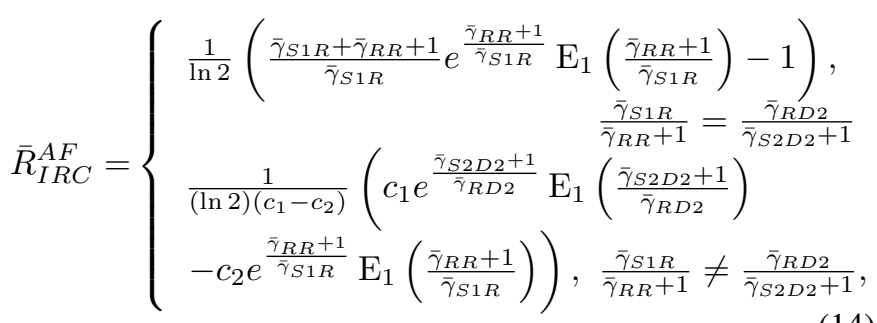

where $\mathrm{E}_{1}(x)=\int_{1}^{\infty} \frac{e^{-x t}}{t} d t$ [20], [40], [44], $c_{1}=$ $\bar{\gamma}_{S 1 R}\left(\bar{\gamma}_{S 2 D 2}+1\right)$, and $c_{2}=\bar{\gamma}_{R D 2}\left(\bar{\gamma}_{R R}+1\right)$.

Proof. See Appendix A.

If each hop of the two-timeslot two-way FD relaying is allocated two orthogonal channels, e.g., using two time slots to transmit data, then it is degraded to HD relaying. Thus, the average rate can be determined from (14) by setting $\bar{\gamma}_{R R}=0$ and $\bar{\gamma}_{S 2 D 2}=0$ and pre-log factor $\frac{1}{2}$. Therefore, the average end-to-end rate for the AF based HD relaying can be written as [39], [40]

$$
\bar{R}_{H D}^{A F}=\left\{\begin{array}{l}
\frac{1}{2 \ln 2}\left[\left(1+\frac{1}{\bar{\gamma}_{S 1 R}}\right) e^{\frac{1}{\bar{\gamma}_{R D 2}}} \mathrm{E}_{1}\left(\frac{1}{\overline{\bar{\gamma}}_{R D 2}}\right)-1\right], \\
\bar{\gamma}_{S 1 R}=\bar{\gamma}_{R D 2} \\
\frac{\bar{\gamma}_{S 1 R} e^{\frac{1}{\bar{\gamma}_{R D 2}}} \mathrm{E}_{1}\left(\frac{1}{\bar{\gamma}_{R D 2}}\right)-\bar{\gamma}_{R D 2} e^{\frac{1}{\bar{\gamma}_{S 1 R}}} \mathrm{E}_{1}\left(\frac{1}{\bar{\gamma}_{S 1 R}}\right)}{(2 \ln 2)\left(\bar{\gamma}_{S 1 R}-\bar{\gamma}_{R D 2}\right)}, \\
\bar{\gamma}_{S 1 R} \neq \bar{\gamma}_{R D 2} .
\end{array}\right.
$$

Note, that compared with (15), the denominator of (14) in Theorem 1 does not contain the constant 2, which indicates that the two-timeslot two-way FD relaying can achieve an extra rate gain due to time multiplexing. However, it also suffers from a certain loss in the rate resulting from residual self-interference, because (14) contains the self-interference terms of $\bar{\gamma}_{R R}$ and $\bar{\gamma}_{S 2 D 2}$. Therefore, if the rate gain from time multiplexing can compensate for the rate loss caused by residual self-interference, the two-timeslot two-way FD relaying can achieve better rate performance than that in the HD case.

An outage occurs when the transmission rate is below the target rate, $R_{t h}$. Thus, the outage probability of the AF based two-timeslot two-way FD $I$-relaying can be expressed as

$$
\begin{aligned}
& P_{\text {out }, I R C}^{A F} \quad \begin{array}{l}
\operatorname{Pr}\left(\log _{2}\left(1+\gamma_{I R C, D 1}^{A F}\right)<R_{t h}\right)+\operatorname{Pr}\left(\log _{2}\left(1+\gamma_{I R C, D 2}^{A F}\right)<R_{t h}\right) \\
2
\end{array} \\
& =\frac{\operatorname{Pr}\left(\gamma_{I R C, D 1}^{A F}<\gamma_{t h}\right)+\operatorname{Pr}\left(\gamma_{I R C, D 2}^{A F}<\gamma_{t h}\right)}{2},
\end{aligned}
$$

where $\gamma_{t h}$ is the SNR threshold for the outage, and we have $\gamma_{t h}=2^{R_{t h}}-1$. The outage probability for the AF based HD relaying can be obtained from (16) by replacing $\gamma_{t h}$ by $2^{2 R_{t h}}-1$. Considering channel reciprocity and identical selfinterference assumption, $P_{\text {out }, I R C}^{A F}$ can be further written as

$$
P_{\text {out }, I R C}^{A F}=\operatorname{Pr}\left(\gamma_{I R C, D 1}^{A F}<\gamma_{t h}\right)=\operatorname{Pr}\left(\gamma_{I R C, D 2}^{A F}<\gamma_{t h}\right)
$$

Note, that the outage probability of the unidirectional link can also be expressed as in (17).
Theorem 2. For a given target rate, $R_{t h}$, the outage probability of the AF based two-timeslot two-way FD I-relaying can be expressed as

$$
\begin{aligned}
P_{\text {out }, I R C}^{A F} & =1-2\left(\frac{\gamma_{t h}\left(\gamma_{t h}+1\right)\left(\bar{\gamma}_{S 2 D 2}+1\right)\left(\bar{\gamma}_{R R}+1\right)}{\bar{\gamma}_{S 1 R} \bar{\gamma}_{R D 2}}\right)^{\frac{1}{2}} \\
& \times e^{-\frac{\gamma_{t h}\left(\bar{\gamma}_{R D 2}\left(\bar{\gamma}_{R R}+1\right)+\bar{\gamma}_{S 1 R}\left(\bar{\gamma}_{S 2 D 2}+1\right)\right)}{\bar{\gamma}_{S 1 R} \bar{\gamma}_{R D 2}}} \\
& \times K_{1}\left(2\left(\frac{\gamma_{t h}\left(\gamma_{t h}+1\right)\left(\bar{\gamma}_{S 2 D 2}+1\right)\left(\bar{\gamma}_{R R}+1\right)}{\bar{\gamma}_{S 1 R} \bar{\gamma}_{R D 2}}\right)^{\frac{1}{2}}\right),
\end{aligned}
$$

where $K_{v}($.$) is the modified Bessel function of the second kind$ [38], and $\gamma_{t h}=2^{R_{t h}}-1$.

\section{Proof. See Appendix B.}

Following similar procedure, the outage probability of the AF based half-duplex relaying can also be determined from (18) by setting $\bar{\gamma}_{R R}=0$ and $\bar{\gamma}_{S 2 D 2}=0$. Therefore, the outage probability can be expressed as [41]

$$
\begin{aligned}
P_{\text {out }, H D}^{A F}= & 1-2\left(\frac{\gamma_{t h}\left(\gamma_{t h}+1\right)}{\bar{\gamma}_{S 1 R} \bar{\gamma}_{R D 2}}\right)^{\frac{1}{2}} e^{-\frac{\gamma_{t h}\left(\bar{\gamma}_{R D 2}+\bar{\gamma}_{S 1 R}\right)}{\bar{\gamma}_{S 1 R} \bar{\gamma}_{R D 2}}} \\
& \times K_{1}\left(2\left(\frac{\gamma_{t h}\left(\gamma_{t h}+1\right)}{\bar{\gamma}_{S 1 R} \bar{\gamma}_{R D 2}}\right)^{\frac{1}{2}}\right) .
\end{aligned}
$$

Note, that for HD and FD relaying, the SNR thresholds for the outage are different. For HD relaying, the SNR threshold for the outage is, $\gamma_{t h}=2^{2 R_{t h}}-1$, while $\gamma_{t h}=2^{R_{t h}}-1$ is used for FD relaying.

2) Decode-and-Forward: In the odd time slots, the relay decodes and buffers the received uplink data from the UE1, then forwards the buffered downlink data from the donor BS to the UE1, while the relay decodes and buffers the received downlink data from the donor BS, then forwards the buffered uplink data from the UE1 to the donor BS in the even time slots.

In the odd time slots, $k=2 n-1, n=1,2,3, \ldots$, the signal transmitted by the relay, $t_{R}[k]$, is

$$
t_{R}[k]=x_{S 2}[k-\tau] .
$$

The instantaneous SNR of the signal received at the relay can be expressed as

$$
\begin{aligned}
\gamma_{S 1 \rightarrow R} & =\frac{\varepsilon\left\{\left|h_{S 1 R}\right|^{2}\left|x_{S 1}[k]\right|^{2}\right\}}{\varepsilon\left\{\left|h_{R R}\right|^{2}\left|t_{R}[k]\right|^{2}\right\}+\varepsilon\left\{\left|n_{R}[k]\right|^{2}\right\}} \\
& =\frac{\left|h_{S 1 R}\right|^{2}}{\left|h_{R R}\right|^{2}+\sigma_{R}^{2}}=\frac{\gamma_{S 1 R}}{\bar{\gamma}_{R R}+1} .
\end{aligned}
$$

By substituting (20) into (3), we have

$$
y_{D 1}[k]=h_{R D 1}\left(x_{S 2}[k-\tau]\right)+h_{S 1 D 1} t_{S 1}[k]+n_{D 1}[k],
$$

The instantaneous SNR of the signal received at the UE1 can be expressed as

$$
\begin{aligned}
\gamma_{I R C, D 1}^{D F} & =\frac{\varepsilon\left\{\left|h_{R D 1}\right|^{2}\left|x_{S 2}[k-\tau]\right|^{2}\right\}}{\varepsilon\left\{\left|h_{S 1 D 1}\right|^{2}\left|t_{S 1}[k]\right|^{2}\right\}+\varepsilon\left\{\left|n_{D 1}[k]\right|^{2}\right\}} \\
& =\frac{\left|h_{R D 1}\right|^{2}}{\left|h_{S 1 D 1}\right|^{2}+\sigma_{D 1}^{2}}=\frac{\gamma_{R D 1}}{\bar{\gamma}_{S 1 D 1}+1} .
\end{aligned}
$$


Similarly, in the even time slots, the instantaneous SNRs of the signals received at the relay and the UE2 can be respectively expressed as

$$
\begin{aligned}
\gamma_{S 2 \rightarrow R} & =\frac{\varepsilon\left\{\left|h_{S 2 R}\right|^{2}\left|x_{S 2}[k]\right|^{2}\right\}}{\varepsilon\left\{\left|h_{R R}\right|^{2}\left|t_{R}[k]\right|^{2}\right\}+\varepsilon\left\{\left|n_{R}[k]\right|^{2}\right\}} \\
& =\frac{\left|h_{S 2 R}\right|^{2}}{\left|h_{R R}\right|^{2}+\sigma_{R}^{2}}=\frac{\gamma_{S 2 R}}{\bar{\gamma}_{R R}+1},
\end{aligned}
$$

and

$$
\begin{aligned}
\gamma_{I R C, D 2}^{D F} & =\frac{\varepsilon\left\{\left|h_{R D 2}\right|^{2}\left|x_{S 1}[k-\tau]\right|^{2}\right\}}{\varepsilon\left\{\left|h_{S 2 D 2}\right|^{2}\left|t_{S 2}[k]\right|^{2}\right\}+\varepsilon\left\{\left|n_{D 2}[k]\right|^{2}\right\}} \\
& =\frac{\left|h_{R D 2}\right|^{2}}{\left|h_{S 2 D 2}\right|^{2}+\sigma_{D 2}^{2}}=\frac{\gamma_{R D 2}}{\bar{\gamma}_{S 2 D 2}+1} .
\end{aligned}
$$

The average end-to-end rate for the DF based two-timeslot two-way FD $I$-relaying is defined as ${ }^{4}$.

$$
\begin{aligned}
\bar{R}_{I R C}^{D F}= & \frac{1}{2}\left(\varepsilon\left\{\log _{2}\left(1+\min \left(\gamma_{S 2 \rightarrow R}, \gamma_{I R C, D 1}^{D F}\right)\right)\right\}\right. \\
& \left.+\varepsilon\left\{\log _{2}\left(1+\min \left(\gamma_{S 1 \rightarrow R}, \gamma_{I R C, D 2}^{D F}\right)\right)\right\}\right) .
\end{aligned}
$$

Considering channel reciprocity and the assumption of identical self-interference, the average rate for the DF based twotimeslot two-way FD $I$-relaying can be further written as

$$
\begin{aligned}
\bar{R}_{I R C}^{D F} & =\varepsilon\left\{\log _{2}\left(1+\min \left(\gamma_{S 2 \rightarrow R}, \gamma_{I R C, D 1}^{D F}\right)\right)\right\} \\
& =\varepsilon\left\{\log _{2}\left(1+\min \left(\gamma_{S 1 \rightarrow R}, \gamma_{I R C, D 2}^{D F}\right)\right)\right\} .
\end{aligned}
$$

Theorem 3. The average end-to-end rate for the DF based two-timeslot two-way FD I-relaying over Rayleigh fading channels, is expressed as

$$
\begin{aligned}
\bar{R}_{I R C}^{D F}= & \frac{1}{\ln 2} e^{\frac{\bar{\gamma}_{S 1 R}\left(\bar{\gamma}_{S 2 D 2}+1\right)+\bar{\gamma}_{R D 2}\left(\bar{\gamma}_{R R}+1\right)}{\bar{\gamma}_{S 1 R} \bar{\gamma}_{R D 2}}} \\
& \times \mathrm{E}_{1}\left(\frac{\bar{\gamma}_{S 1 R}\left(\bar{\gamma}_{S 2 D 2}+1\right)+\bar{\gamma}_{R D 2}\left(\bar{\gamma}_{R R}+1\right)}{\bar{\gamma}_{S 1 R} \bar{\gamma}_{R D 2}}\right) .
\end{aligned}
$$

Proof. See Appendix C.

In the same way, the average end-to-end rate for the DF based HD relaying over Rayleigh fading channels, can be determined from (28) by setting $\bar{\gamma}_{R R}=0$ and $\bar{\gamma}_{S 2 D 2}=0$ and pre-log factor $\frac{1}{2}$. Therefore,

$$
\bar{R}_{H D}^{D F}=\frac{1}{2 \ln 2} e^{\frac{\bar{\gamma}_{S 1 R}+\bar{\gamma}_{R D 2}}{\bar{\gamma}_{S 1 R} \bar{\gamma}_{R D 2}}} \mathrm{E}_{1}\left(\frac{\bar{\gamma}_{S 1 R}+\bar{\gamma}_{R D 2}}{\bar{\gamma}_{S 1 R} \bar{\gamma}_{R D 2}}\right) \text {. }
$$

Note, that in DF protocol, a similar trend in the rate performance can be found as the AF one. However, the effect of residual self-interference on DF protocol is less than that of the AF one, because DF protocol suppresses residual selfinterference propagation, while the last one propagates residual self-interference, which is indicated through the comparison of the instantaneous SNRs in (11) and (25).

The outage probability of the DF based two-timeslot twoway FD $I$-relaying, is defined as

$$
\begin{aligned}
P_{\text {out }, I R C}^{D F}= & \frac{1}{2}\left(\operatorname{Pr}\left(\min \left(\gamma_{S 2 \rightarrow R}, \gamma_{I R C, D 1}^{D F}\right)<\gamma_{t h}\right)\right. \\
& \left.+\operatorname{Pr}\left(\min \left(\gamma_{S 1 \rightarrow R}, \gamma_{I R C, D 2}^{D F}\right)<\gamma_{t h}\right)\right) .
\end{aligned}
$$

${ }^{4}$ Note, that achieving the average rate, $\min \left(\varepsilon\left\{R_{R}\right\}, \varepsilon\left\{R_{D}\right\}\right)$ [11], requires an idealistic setup with an infinite buffer at the relay, which can avoid overflows and underflows but suffer from large end-to-end delay.
Considering channel reciprocity and identical self-interference assumption, $P_{\text {out }, I R C}^{D F}$ can be further expressed as

$$
\begin{aligned}
P_{\text {out }, \text { IRC }}^{D F}= & 1-\left(1-\operatorname{Pr}\left(\gamma_{S 2 \rightarrow R}<\gamma_{t h}\right)\right) \\
& \times\left(1-\operatorname{Pr}\left(\gamma_{I R C, D 1}^{D F}<\gamma_{t h}\right)\right) \\
= & 1-\left(1-\operatorname{Pr}\left(\gamma_{S 1 \rightarrow R}<\gamma_{t h}\right)\right) \\
& \times\left(1-\operatorname{Pr}\left(\gamma_{I R C, D 2}^{D F}<\gamma_{t h}\right)\right) .
\end{aligned}
$$

Theorem 4. For a given target rate, $R_{t h}$, the outage probability of the DF based two-timeslot two-way FD I-relaying is given by

$$
P_{\text {out }, I R C}^{D F}=1-e^{\frac{-\gamma_{t h}\left(\bar{\gamma}_{S 1 R}\left(\bar{\gamma}_{S 2 D 2}+1\right)+\bar{\gamma}_{R D 2}\left(\bar{\gamma}_{R R}+1\right)\right)}{\bar{\gamma}_{S 1 R} \bar{\gamma}_{R D 2}}},
$$

where $\gamma_{t h}=2^{R_{t h}}-1$.

Proof. See Appendix D.

For the same reason, the outage probability of the DF based HD relaying can be determined from (32) by setting $\bar{\gamma}_{R R}=0$ and $\bar{\gamma}_{S 2 D 2}=0$. Thus, the outage probability can be written as [41]

$$
P_{\text {out }, H D}^{D F}=1-e^{\frac{-\gamma_{t h}\left(\bar{\gamma}_{S 1 R}+\bar{\gamma}_{R D 2}\right)}{\bar{\gamma}_{S 1 R} \bar{\gamma}_{R D 2}}} .
$$

Note, that for HD and FD relaying, the SNR thresholds for the outage are different. For HD relaying, the SNR threshold for the outage is, $\gamma_{t h}=2^{2 R_{t h}}-1$, while $\gamma_{t h}=2^{R_{t h}}-1$ is used for FD relaying.

\section{B. Y-Relaying Channel}

For $Y$-relaying channel, in the odd time slots, the relay receives the uplink data from the UE1 and forwards the buffered downlink data to the UE2 on the same carrier frequency, simultaneously. Note, that the relay suffers from residual self-interference due to the co-channel transmitted signal and imperfect interference cancellation, while the UE2 suffers from inter-user interference because the UE1 transmits signal on the same carrier frequency, simultaneously. In the even time slots, simultaneous bidirectional data exchange is achieved between the relay and the donor BS.

1) Amplify-and-Forward: In the odd time slots, the relay receives and buffers uplink data from the UE1, then forwards the buffered downlink data to the UE2 by amplifying the signal, based on the channel gains, while the UE2 receives downlink data forwarded by the relay and inter-user interference from the UE1. In the even time slots, the relay receives and buffers downlink data from the donor BS, then forwards the buffered uplink data to the donor BS by amplifying the signal, based on the relaying channel gains.

Compared with the $I$-relaying channel, in the $Y$-relaying channel, the destination UE suffers from inter-user interference, which is due to other UEs' co-channel uplink data transmission, instead of residual self-interference. Therefore, the instantaneous SNRs at the destination node UE2, can be expressed as

$\gamma_{Y R C, D 1}^{A F}=\frac{\gamma_{R D 1} \gamma_{S 2 R}}{\gamma_{R D 1}\left(\bar{\gamma}_{R R}+1\right)+\left(\gamma_{S 1 D 1}+1\right)\left(\gamma_{S 2 R}+\bar{\gamma}_{R R}+1\right)}$ 
while the instantaneous SNRs at the destination donor BS is written as in (11). Note, that comparing (34) with (10), $\gamma_{S 1 D 1}$ is the main difference between the $Y$ and the $I$ cases, where instead of $\gamma_{S 1 D 1}$ we have $\bar{\gamma}_{S 1 D 1}$.

Theorem 5. For the $S 1 \rightarrow R \rightarrow D 2$ link, the average endto-end rate for the AF based two-timeslot two-way FD $Y$ relaying over Rayleigh fading channels, is expressed as in (14). For the $S 2 \rightarrow R \rightarrow D 1$ link, the average end-to-end rate can be upper bounded as in (35), shown at the bottom of the next page, where $F_{1}(x)=e^{x} \mathrm{E}_{1}(x), c_{3}=$ $\bar{\gamma}_{R D 1}\left(\bar{\gamma}_{R R}+1\right), c_{4}=\bar{\gamma}_{S 2 R}\left(\bar{\gamma}_{S 1 D 1}+1\right), c_{5}=\frac{\bar{\gamma}_{R D 1}}{\bar{\gamma}_{R D 1}-\bar{\gamma}_{S 1 D 1}}$, $C_{1}:\left\{\bar{\gamma}_{R D 1}=\bar{\gamma}_{S 1 D 1}\right.$ and $\left.\frac{\bar{\gamma}_{S 2 R}}{\bar{\gamma}_{R R+1}}=\frac{\bar{\gamma}_{R D 1}}{\bar{\gamma}_{S 1 D 1}+1}\right\}, C_{2}$ : $\left\{\bar{\gamma}_{R D 1}=\bar{\gamma}_{S 1 D 1}\right.$ and $\left.\frac{\bar{\gamma}_{S 2 R}}{\bar{\gamma}_{R R}+1} \neq \frac{\bar{\gamma}_{R D 1}}{\bar{\gamma}_{S 1 D 1}+1}\right\}, C_{3}:\left\{\bar{\gamma}_{R D 1} \neq\right.$ $\bar{\gamma}_{S 1 D 1}$ and $\left.\frac{\bar{\gamma}_{S 2 R}}{\bar{\gamma}_{R R}+1}=\frac{\bar{\gamma}_{R D 1}}{\bar{\gamma}_{S 1 D 1+1}}\right\}$, and $C_{4}:\left\{\bar{\gamma}_{R D 1} \neq\right.$ $\bar{\gamma}_{S 1 D 1}$ and $\left.\frac{\bar{\gamma}_{S 2 R}}{\bar{\gamma}_{R R}+1} \neq \frac{\bar{\gamma}_{R D 1}}{\bar{\gamma}_{S 1 D 1}+1}\right\}$.

Proof. See Appendix E.

Note, that if there is no inter-user interference, according to (14), by substituting $\bar{\gamma}_{S 2 R}$ and $\bar{\gamma}_{R D 1}$ for $\bar{\gamma}_{S 1 R}$ and $\bar{\gamma}_{R D 2}$, respectively, and removing $\bar{\gamma}_{S 2 D 2}$, the average end-to-end rate for the $S 2 \rightarrow R \rightarrow D 1$ link is

$$
\bar{R}_{Y R C, D 1}^{A F}=\left\{\begin{array}{r}
\frac{1}{\ln 2}\left(\frac{\bar{\gamma}_{S 2 R}+\bar{\gamma}_{R R}+1}{\bar{\gamma}_{S 2 R}} e^{\frac{\bar{\gamma}_{R R}+1}{\bar{\gamma}_{S 2 R}}} \mathrm{E}_{1}\left(\frac{\bar{\gamma}_{R R}+1}{\bar{\gamma}_{S 2 R}}\right)-1\right) \\
\frac{\bar{\gamma}_{S 2 R}}{\bar{\gamma}_{R R}+1}=\bar{\gamma}_{R D 1} \\
\frac{\bar{\gamma}_{S 2 R} e^{\frac{1}{\bar{\gamma}_{R D 1}}} \mathrm{E}_{1}\left(\frac{1}{\bar{\gamma}_{R D 1}}\right)-c_{3} e^{\frac{\bar{\gamma}_{R R}+1}{\bar{\gamma}_{S 2 R}}} \mathrm{E}_{1}\left(\frac{\bar{\gamma}_{R R}+1}{\bar{\gamma}_{S 2 R}}\right)}{(\ln 2)\left(\bar{\gamma}_{S 2 R}-c_{3}\right)} \\
\frac{\bar{\gamma}_{S 2 R}}{\bar{\gamma}_{R R}+1} \neq \bar{\gamma}_{R D 1}
\end{array}\right.
$$

Theorem 6. For a given target rate, $R_{t h}$, the outage probability of the $S 1 \rightarrow R \rightarrow D 2$ link in the $A F$ based two-timeslot two-way FD $Y$-relaying can be formulated as in (18), while the outage probability of the $S 2 \rightarrow R \rightarrow D 1$ link can be upper bounded as

$$
\begin{aligned}
& P_{\text {out }, D 1}^{Y R C, A F} \\
& \quad \leq 1-2\left(\frac{\gamma_{t h}\left(\gamma_{t h}+1\right)\left(\bar{\gamma}_{R R}+1\right)\left(\bar{\gamma}_{S 1 D 1}+1\right)}{\bar{\gamma}_{S 2 R} \bar{\gamma}_{R D 1}}\right)^{\frac{1}{2}} \\
& \quad \times e^{-\frac{\gamma_{t h}\left(\bar{\gamma}_{R D 1}\left(\bar{\gamma}_{R R}+1\right)+\bar{\gamma}_{S 2 R}\left(\bar{\gamma}_{S 1 D 1}+1\right)\right)}{\bar{\gamma}_{S 2 R} \bar{\gamma}_{R D 1}}} \\
& \quad \times K_{1}\left(2\left(\frac{\gamma_{t h}\left(\gamma_{t h}+1\right)\left(\bar{\gamma}_{R R}+1\right)\left(\bar{\gamma}_{S 1 D 1}+1\right)}{\bar{\gamma}_{S 2 R} \bar{\gamma}_{R D 1}}\right)^{\frac{1}{2}}\right),
\end{aligned}
$$

where $\gamma_{t h}=2^{R_{t h}}-1$.

Proof. See Appendix F.

Note, that if there is no inter-user interference between two UEs, $P_{\text {out }, D 1}^{Y R C, A F}$ can be further simplified as

$$
\begin{aligned}
P_{\text {out }, D 1}^{Y R C, A F}= & 1-2\left(\frac{\gamma_{t h}\left(\gamma_{t h}+1\right)\left(\bar{\gamma}_{R R}+1\right)}{\bar{\gamma}_{S 2 R} \bar{\gamma}_{R D 1}}\right)^{\frac{1}{2}} \\
& \times e^{-\frac{\gamma_{t h}\left(\bar{\gamma}_{R D 1}\left(\bar{\gamma}_{R R}+1\right)+\bar{\gamma}_{S 2 R}\right)}{\bar{\gamma}_{S 2 R} \bar{\gamma}_{R D 1}}} \\
& \times K_{1}\left(2\left(\frac{\gamma_{t h}\left(\gamma_{t h}+1\right)\left(\bar{\gamma}_{R R}+1\right)}{\bar{\gamma}_{S 2 R} \bar{\gamma}_{R D 1}}\right)^{\frac{1}{2}}\right) .
\end{aligned}
$$

2) Decode-and-Forward: In the odd time slots, the relay decodes and buffers the received uplink data from the UE1, and forwards the buffered downlink data to the UE2, while the UE2 receives downlink data forwarded by the relay and inter-user interference from the UE1. In the even time slots, the relay decodes and buffers the received downlink data from the donor BS, and forwards the buffered uplink data to the donor BS.

Considering $\gamma_{S 1 D 1}$ as inter-user interference, the instantaneous SNRs of the DF based two-timeslot two-way FD $Y$ relaying at two destination nodes, can be obtained from the corresponding $I$-relaying one. The instantaneous SNR at the destination UE2 is expressed as

$$
\gamma_{Y R C, D 1}^{D F}=\frac{\gamma_{R D 1}}{\gamma_{S 1 D 1}+1} .
$$

The instantaneous SNR at the destination donor BS can be written as in (25).

Theorem 7. For the $S 1 \rightarrow R \rightarrow D 2$ link, the average end-to-end rate for the DF based two-timeslot two-way FD $Y$-relaying over Rayleigh fading channels, can be expressed as in (28), while for the $S 2 \rightarrow R \rightarrow D 1$ link, the average end-to-end rate is lower bounded as

$$
\begin{aligned}
\bar{R}_{Y R C, D 1}^{D F} \geq & \frac{1}{\ln 2} e^{\frac{\bar{\gamma}_{S 2 R}\left(\bar{\gamma}_{S 1 D 1}+1\right)+\bar{\gamma}_{R D 1}\left(\bar{\gamma}_{R R}+1\right)}{\bar{\gamma}_{S 2 R} \bar{\gamma}_{R D 1}}} \\
& \times \mathrm{E}_{1}\left(\frac{\bar{\gamma}_{S 2 R}\left(\bar{\gamma}_{S 1 D 1}+1\right)+\bar{\gamma}_{R D 1}\left(\bar{\gamma}_{R R}+1\right)}{\bar{\gamma}_{S 2 R} \bar{\gamma}_{R D 1}}\right) .
\end{aligned}
$$

Proof. See Appendix G.

Theorem 8. For a given target rate, $R_{t h}$, the outage probability of the $S 1 \rightarrow R \rightarrow D 2$ link in the DF based two-timeslot twoway FD $Y$-relaying is expressed as in (32), while the outage probability of the $S 2 \rightarrow R \rightarrow D 1$ link can be formulated as

$$
P_{\text {out }, D 1}^{Y R C, D F}=1-\frac{\bar{\gamma}_{R D 1}}{\gamma_{t h} \bar{\gamma}_{S 1 D 1}+\bar{\gamma}_{R D 1}} e^{-\frac{\gamma_{t h}\left(\bar{\gamma}_{S 2 R}+\bar{\gamma}_{R D 1}\left(\bar{\gamma}_{R R}+1\right)\right)}{\bar{\gamma}_{S 2 R} \bar{\gamma}_{R D 1}}},
$$

where $\gamma_{t h}=2^{R_{t h}}-1$.

Proof. See Appendix H.

\section{Numerical Results, Simulations AND DISCUSSION}

In this section, numerical results for the average end-toend rate and the outage probability of the two-timeslot twoway FD relaying scheme are presented, together with Monte Carlo simulations. We consider that the average SNR of the access link is the same with that of the backhaul link, and also for both inter-user interference and residual self-interference. In simulations, we first fix each node's transmit power, then adjust the distance and location between two nodes, so that we can change the average SNRs of their end-to-end link.

Fig. 3(a) and Fig. 3(b) compare the average end-to-end rate for the $\mathrm{AF}$ and $\mathrm{DF}$ based two-timeslot two-way FD $I$ and HD relaying over Rayleigh fading channels, respectively. The results clearly show that the two-timeslot two-way FD $I$-relaying can achieve higher average rate than HD relaying, but double rate cannot be obtained. In addition, there is a 


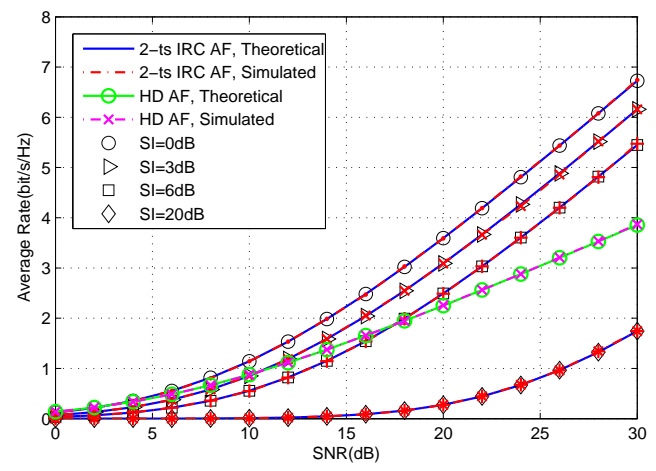

(a)

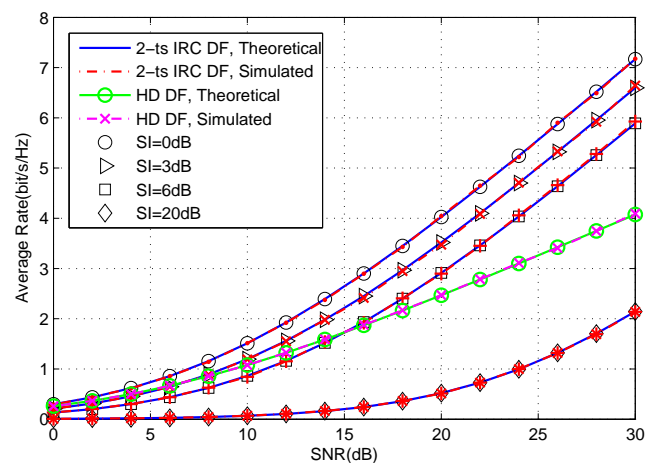

(b)

Fig. 3. Average rate for two-timeslot two-way FD $I$ - and HD relaying: a) AF protocol; b) DF protocol

cross point between the two curves, and this point shifts to the right with the increase in residual self-interference. This is because FD operation can achieve an extra rate gain by time multiplexing, but it also suffers from residual self-interference, which results in a loss in the rate performance. With the increase of residual self-interference, the rate gain from time multiplexing cannot compensate for the rate loss, caused by the residual self-interference. It is also shown that DF protocol can achieve higher average rate than the AF one, because AF relaying propagates residual self-interference, while the DF one can suppress this propagation.

Fig. 4(a) and Fig. 4(b) depict the outage probability of the AF and DF based two-timeslot two-way FD $I$ - and HD relaying with $R_{t h}=1 \mathrm{~b} / \mathrm{s} / \mathrm{Hz}$, respectively. The results show that the outage performance of the two-timeslot two-way FD $I$-relaying is better than that in HD relaying, when residual self-interference is below a certain level (e.g. $<3 \mathrm{~dB})$. DF protocol can achieve better outage performance than the AF

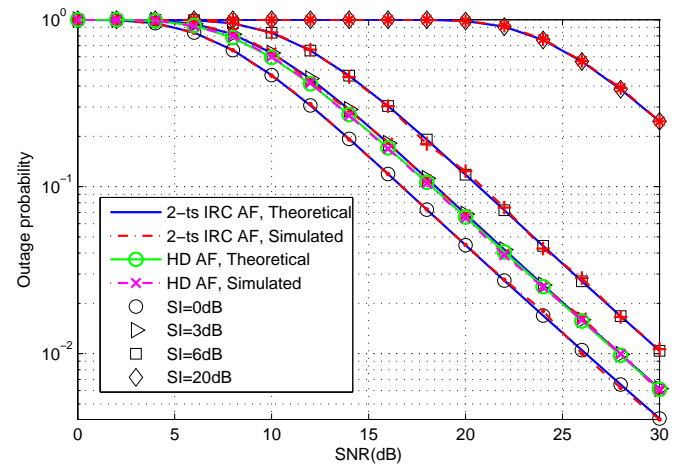

(a)

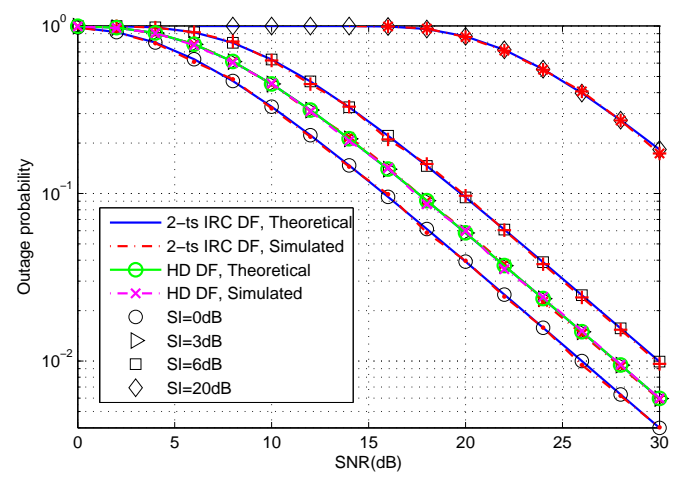

(b)

Fig. 4. Outage probability of the two-timeslot two-way FD $I$ - and HD relaying: a) AF protocol; b) DF protocol

one in two-timeslot two-way FD $I$-relaying.

Fig. 5(a) shows the average rate for the unidirectional links in the AF based two-timeslot two-way FD $Y$-relaying. Fig. 5(b) compares the average rate for the AF based two-timeslot two-way FD $Y$ - and HD relaying. The results are similar as those in corresponding $I$-relaying shown in Fig. 3, but the average rate for the downlink (i. e., $S 2 \rightarrow R \rightarrow D 1$ link) is slightly better than that in uplink, when self-interference is very severe. This is because inter-user interference is assumed to be Rayleigh fading, while residual self-interference is free of fading. Thus, the effect of inter-user interference on the rate is less than that of residual self-interference, under the same average SNRs.

Fig. 6(a) depicts the outage probability of the unidirectional links in the AF based two-timeslot two-way FD $Y$-relaying. Fig. 6(b) depicts the outage probability of the AF two-timeslot two-way FD $Y$ - and HD relaying with $R_{t h}=1 \mathrm{~b} / \mathrm{s} / \mathrm{Hz}$. Similar results can be obtained as those in $I$-relaying shown in Fig.

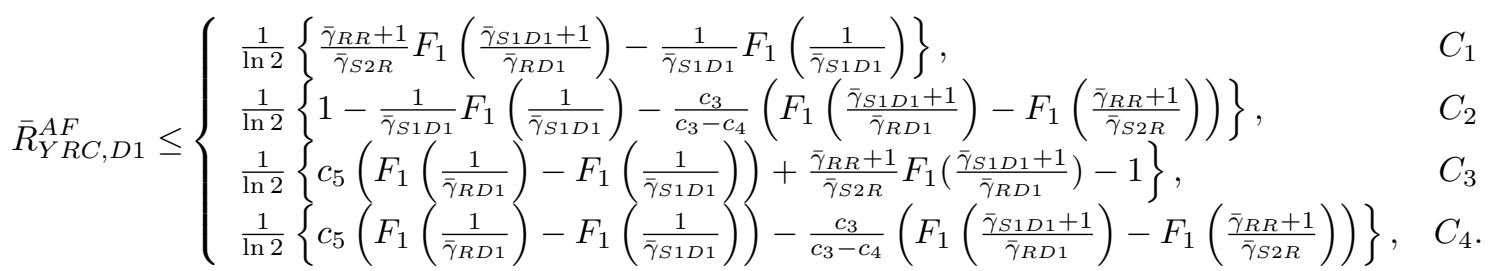




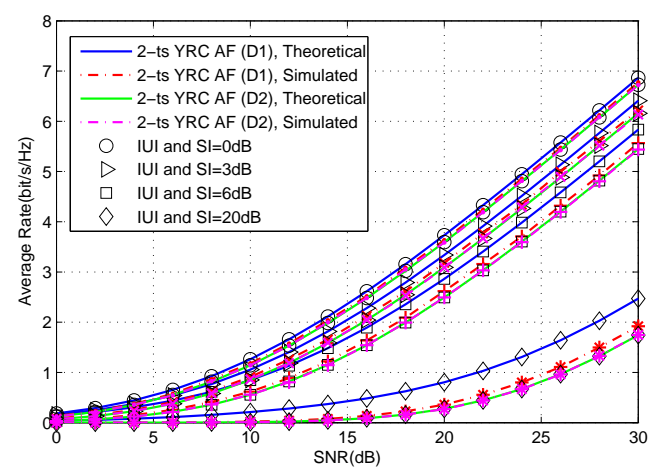

(a)

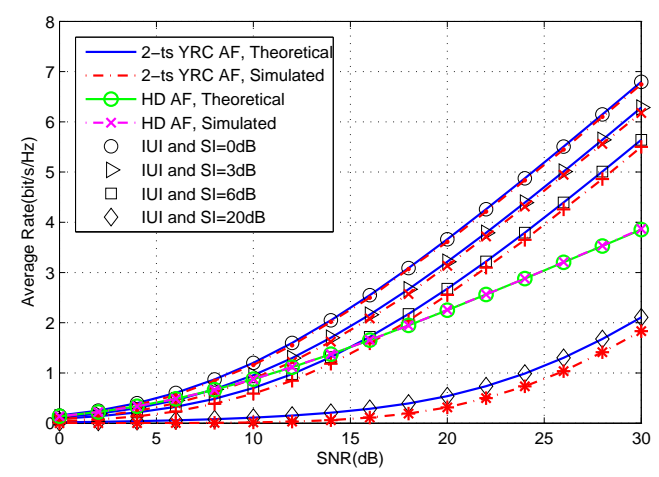

(b)

Fig. 5. Average rate for the AF two-timeslot two-way FD $Y$ - and HD relaying: a) 2-ts YRC $D_{1}$ and $D_{2}$; b) 2-ts YRC vs. HD relaying

4 , but the outage probability of the downlink is slightly better than that in uplink, when self-interference is very severe.

Fig. 7(a) illustrates the average end-to-end rate for the unidirectional links in the DF based two-timeslot two-way FD $Y$-relaying. Fig. 7(b) compares the average end-to-end rate for the DF two-timeslot two-way FD $Y$-relaying and HD relaying. The results are similar as those in the AF one shown in Fig. 5.

Fig. 8(a) depicts the outage probability of the unidirectional links in the DF based two-timeslot two-way FD $Y$-relaying. Fig. 8(b) depicts the outage probability of the DF based twotimeslot two-way FD $Y$ - and HD relaying with $R_{t h}=1$ $\mathrm{b} / \mathrm{s} / \mathrm{Hz}$. Similar results can be obtained as those in the AF one shown in Fig. 6.

Fig. 9 compares the average end-to-end rate for the AF and DF based two-timeslot two-way FD $I$-, $Y$-, and HD relaying. The results demonstrate that two rate curves of two-timeslot two-way FD $I$ - and $Y$-relaying are basically overlapped, when the average SNRs of inter-user interference is same as self-interference. Compared with two-timeslot twoway FD $I$-relaying, $Y$-relaying can achieve a slight rate gain, only when the average SNRs of inter-user interference and self-interference are at very high region.

Fig. 10 depicts the outage probability of the AF and DF based the two-timeslot two-way FD $I$-, $Y$-, and HD relaying with $R_{t h}=1 \mathrm{~b} / \mathrm{s} / \mathrm{Hz}$. Similar results can be obtained as the rate performance shown in Fig. 9.

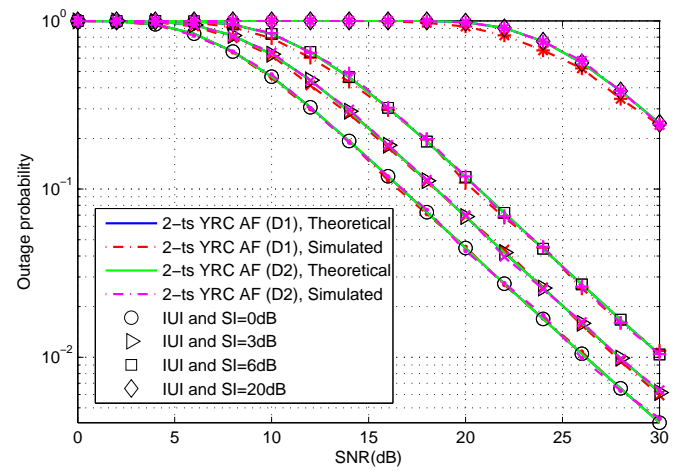

(a)

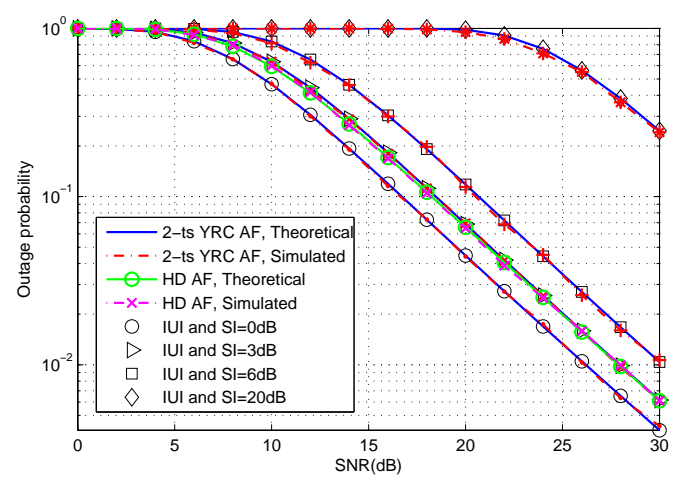

(b)

Fig. 6. Outage probability of the AF two-timeslot two-way FD $Y$ - and HD relaying: a) 2-ts YRC $D_{1}$ and $D_{2}$; b) 2-ts YRC vs. HD relaying

\section{Conclusions}

A two-timeslot two-way FD relaying scheme, which was based on dividing the access link and the backhaul link in time domain, was proposed and investigated. Closed-form expressions for the average end-to-end rate and the outage probability, were derived. According to UE capability and services, two scenarios of $I$ - and $Y$-relaying channels, were considered. The results clearly showed that the proposed scheme could achieve higher rate and better outage performance than HD relaying, when residual self-interference was below a certain level. It was also shown that DF protocol could achieve higher rate than that in the AF one in the proposed relaying scheme. In addition, the effect of inter-user interference on the average rate and the outage probability was slight weaker than self-interference, when considering that inter-user interference is Rayleigh fading and self-interference is non-fading. Furthermore, there were tradeoffs between FD and HD modes, $I$ - and $Y$-relaying schemes. If residual selfinterference was not very severe, FD mode should be adopted, while $Y$-relaying scheme with weak inter-user interference was superior to $I$-relaying scheme, if UE could not suppress residual self-interference very well. In summary, the twotimeslot two-way FD relaying scheme can achieve reasonable tradeoff of performance and complexity, thus it will be a potentially efficient solution for $5 \mathrm{G}$ systems. In a future work, the effect of the access/backhaul link time slot configuration on the proposed scheme, will be investigated. 


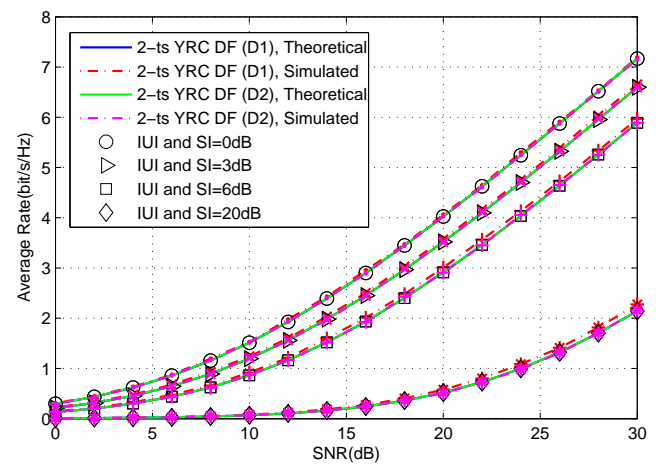

(a)

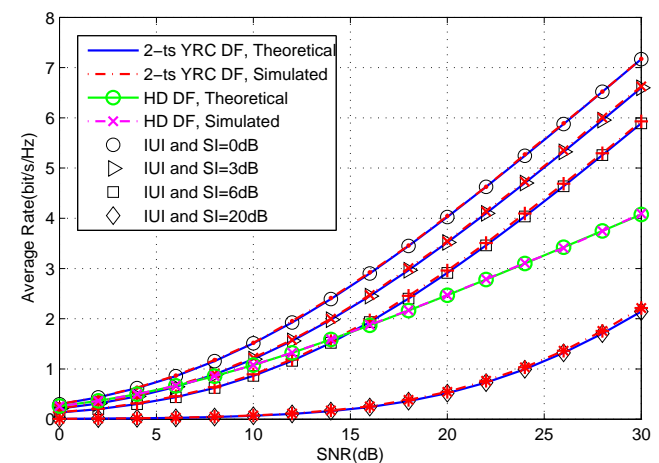

(b)

Fig. 7. Average rate for the DF two-timeslot two-way FD $Y$ - and HD relaying: a) 2-ts YRC $D_{1}$ and $D_{2}$; b) 2-ts YRC vs. HD relaying

\section{APPENDIX A}

\section{PROOF OF THEOREM 1}

Combining (11) and (13), the average rate for the AF based two-timeslot two-way FD $I$-relaying over Rayleigh fading channels is written as in (42), shown at the bottom of the page.

Since [40, eq. (12)] is the integral result of [40, eq. (8)] and (42) has the similar form as [40, eq. (8)], applying [40, eq. (12)], the average rate can be expressed as in (43), shown at the bottom of the next page. After simplifying (43), $\bar{R}_{I R C}^{A F}$ can be obtained as in (14) and the proof is completed.

\section{APPENDIX B \\ PROOF OF THEOREM 2}

For the AF based two-timeslot two-way FD $I$-relaying, the instantaneous SNR of the signal received at the donor BS can be rewritten as

$$
\begin{aligned}
\gamma_{I R C}^{A F} & =\frac{\frac{\gamma_{S 1 R}}{\bar{\gamma}_{R R}+1} \frac{\gamma_{R D 2}}{\bar{\gamma}_{S 2 D 2}+1}}{\frac{\gamma_{S 1 R}}{\bar{\gamma}_{R R}+1}+\frac{\gamma_{R D 2}}{\bar{\gamma}_{S 2 D 2}+1}+1} . \\
\bar{R}_{I R C}^{A F} & =\varepsilon\left\{\log _{2}\left(1+\frac{\gamma_{R D 2} \gamma_{S 1 R}}{\gamma_{R D 2}\left(\bar{\gamma}_{R R}+1\right)+\left(\bar{\gamma}_{S 2 D 2}+1\right)\left(\gamma_{S 1 R}+\bar{\gamma}_{R R}+1\right)}\right)\right\} \\
& =\varepsilon\left\{\log _{2}\left(1+\frac{\gamma_{S 1 R}}{\bar{\gamma}_{R R}+1}\right)+\log _{2}\left(1+\frac{\gamma_{R D 2}}{\bar{\gamma}_{S 2 D 2}+1}\right)-\log _{2}\left(1+\frac{\gamma_{S 1 R}}{\bar{\gamma}_{R R}+1}+\frac{\gamma_{R D 2}}{\bar{\gamma}_{S 2 D 2}+1}\right)\right\} .
\end{aligned}
$$

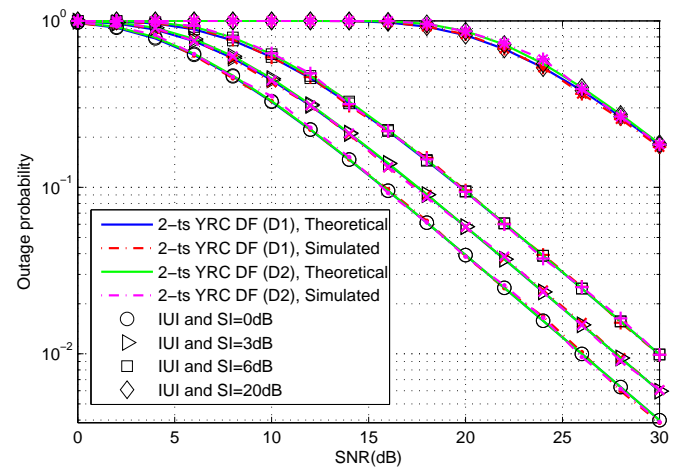

(a)

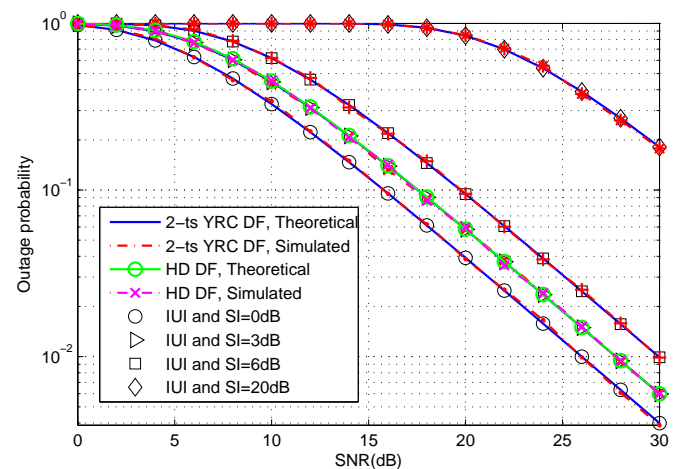

(b)

Fig. 8. Outage probability of the DF based two-timeslot two-way FD $Y$ - and HD relaying: a) 2-ts YRC $D_{1}$ and $D_{2}$; b) 2-ts YRC vs. HD relaying

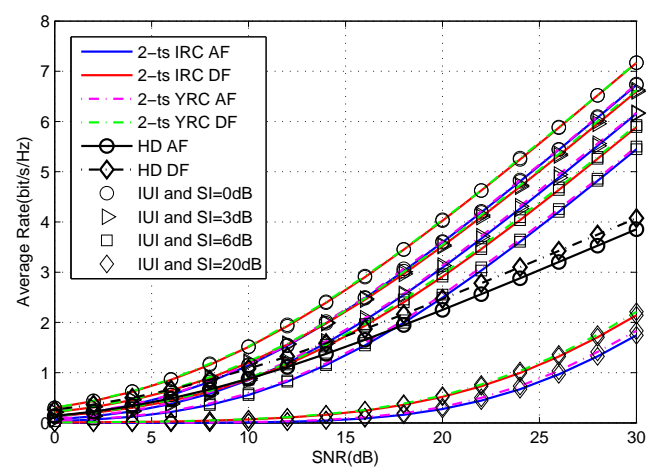

Fig. 9. Average rate for the AF and DF based two-timeslot two-way FD $I$ $Y-$, and HD relaying 


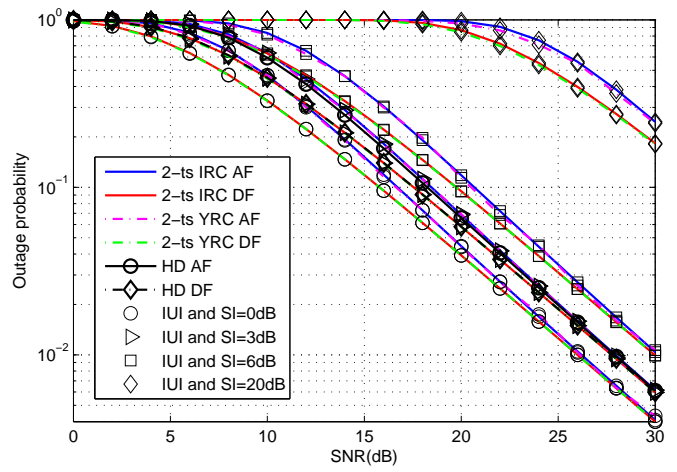

Fig. 10. Outage probability of the AF and DF based two-timeslot two-way FD $I-, Y-$, and HD relaying

probability can be expressed as

$$
\begin{aligned}
P_{\text {out }}^{I R C, A F}= & -2\left(\frac{\gamma_{t h}\left(\gamma_{t h}+1\right)}{\left.\bar{\gamma}_{S 1 R} \frac{\bar{\gamma}_{R D 2}}{\bar{\gamma}_{R R}+1}\right)^{\frac{1}{2}} e^{2}} e^{-\gamma_{t h}\left(\frac{\bar{\gamma}_{R R}+1}{\bar{\gamma}_{S 1 R}}+\frac{\bar{\gamma}_{S 2 D 2}+1}{\bar{\gamma}_{R D 2}}\right)}\right. \\
& \times K_{1}\left(2\left(\frac{\gamma_{t h}\left(\gamma_{t h}+1\right)}{\left.\left.\frac{\overline{\bar{\gamma}}_{S 1 R} \overline{\bar{\gamma}}_{R D 2}}{\bar{\gamma}_{R R}+1}\right)^{\frac{1}{2}}\right)}\right)\right.
\end{aligned}
$$

Finally, simplifying (45), the outage probability can be written as in (18) and the proof is completed.

\section{APPENDIX C \\ Proof of Theorem 3}

We first define a random variable $w$ and its PDF is, $f(w)=\left(\frac{\bar{\gamma}_{R R}+1}{\bar{\gamma}_{S 1 R}}+\frac{\bar{\gamma}_{S 2 D 2}+1}{\bar{\gamma}_{R D 2}}\right) e^{-\left(\frac{\bar{\gamma}_{R R}+1}{\bar{\gamma}_{S 1 R}}+\frac{\bar{\gamma}_{S 2 D 2}+1}{\bar{\gamma}_{R D 2}}\right) w}$. According to (21), (25), and (27), the average rate for the DF based two-timeslot two-way FD $I$-relaying can be rewritten as

$$
\begin{aligned}
\bar{R}_{I R C}^{D F}= & \int_{0}^{\infty} \log _{2}(1+w) f(w) d w \\
= & \int_{0}^{\infty} \log _{2}(1+w)\left(\frac{\bar{\gamma}_{R R}+1}{\bar{\gamma}_{S 1 R}}+\frac{\bar{\gamma}_{S 2 D 2}+1}{\bar{\gamma}_{R D 2}}\right) \\
& \times e^{-\left(\frac{\bar{\gamma}_{R R}+1}{\bar{\gamma}_{S 1 R}}+\frac{\bar{\gamma}_{S 2 D 2}+1}{\bar{\gamma}_{R D 2}}\right) w} d w \\
= & \int_{0}^{\infty} \frac{1}{\ln 2} \ln (1+w)\left(\frac{\bar{\gamma}_{R R}+1}{\bar{\gamma}_{S 1 R}}+\frac{\bar{\gamma}_{S 2 D 2}+1}{\bar{\gamma}_{R D 2}}\right) \\
& \times e^{-\left(\frac{\bar{\gamma}_{R R}+1}{\bar{\gamma}_{S 1 R}}+\frac{\bar{\gamma}_{S 2 D 2}+1}{\bar{\gamma}_{R D 2}}\right) w} d w .
\end{aligned}
$$

Finally, according to [42, eq. (19b)], the average rate can be written as in (28) and the proof is completed.

\section{APPENDIX D \\ PROOF OF THEOREM 4}

Combining (21), (25), and (31), the outage probability of the DF based two-timeslot two-way FD $I$-relaying can be expressed as

$$
\begin{aligned}
P_{\text {out }, \text { IRC }}^{D F}= & -\left(1-\int_{0}^{\gamma_{t h}\left(\bar{\gamma}_{R R}+1\right)} \frac{1}{\bar{\gamma}_{S 1 R}} e^{-\frac{x}{\bar{\gamma}_{S 1 R}}} d x\right) \\
& \times\left(1-\int_{0}^{\gamma_{t h}\left(\bar{\gamma}_{S 2 D 2}+1\right)} \frac{1}{\bar{\gamma}_{R D 2}} e^{-\frac{y}{\bar{\gamma}_{R D 2}}} d y\right) \\
= & -\left(\int_{\gamma_{t h}\left(\bar{\gamma}_{R R}+1\right)}^{\infty} \frac{1}{\bar{\gamma}_{S 1 R}} e^{-\frac{x}{\bar{\gamma}_{S 1 R}}} d x\right) \\
& \times\left(\int_{\gamma_{t h}\left(\bar{\gamma}_{S 2 D 2}+1\right)}^{\infty} \frac{1}{\bar{\gamma}_{R D 2}} e^{-\frac{y}{\bar{\gamma}_{R D 2}}} d y\right) .
\end{aligned}
$$

Substituting the corresponding parameters in [41, eq. (21)] by the suitable values, the outage probability can be written as in (32) and the proof is completed.

\section{APPENDIX E}

\section{PROOF OF THEOREM 5}

For the $S 1 \rightarrow R \rightarrow D 2$ link, the average rate for the $\mathrm{AF}$ two-timeslot two-way FD $Y$-relaying over Rayleigh fading channels is written as in (14), while for the $S 2 \rightarrow R \rightarrow D 1$ link, the average rate, $\bar{R}_{Y R C, D 1}^{A F}$, has the similar form as in (13), thus, replacing $\gamma_{I R C, D 1}^{A F}$ in (13) by $\gamma_{Y R C, D 1}^{A F}$ in (34), the average rate is expressed as in (48), shown at the bottom of the next page. Function $g(x, y, z)$ in (48) is, $g(x, y, z)=\frac{e^{-x / \bar{\gamma}_{R D 1}}}{\bar{\gamma}_{R D 1}} \frac{e^{-y / \bar{\gamma}_{S 2 R}}}{\bar{\gamma}_{S 2 R}} \frac{e^{-z / \bar{\gamma}_{S 1 D 1}}}{\bar{\gamma}_{S 1 D 1}}$. Through merging the same items and integrating, the triple integral, $I_{5,1}$, can be rewritten as

$$
\begin{aligned}
I_{5,1}= & \underbrace{\int_{0}^{\infty} \ln \left(y+\bar{\gamma}_{R R}+1\right) \frac{e^{-y / \bar{\gamma}_{S 2 R}}}{\bar{\gamma}_{S 2 R}} d y}_{I_{5,1-1}} \\
& +\underbrace{\int_{0}^{\infty} \int_{0}^{\infty} \ln (x+z+1) \frac{e^{-x / \bar{\gamma}_{R D 1}}}{\bar{\gamma}_{R D 1}} \frac{e^{-z / \bar{\gamma}_{S 1 D 1}}}{\bar{\gamma}_{S 1 D 1}} d x d z}_{I_{5,1-2}}
\end{aligned}
$$

The integrals $I_{5,1-1}$ and $I_{5,1-2}$ can be respectively solved as follows. [43, eq. (2.6.23.5)] gives the result of integral form $\int_{0}^{\infty} e^{-p x} \ln (a+b x) d x$, where $x$ is variable of integration; and $a, b$, and $p$ are constants. Since the first part, $I_{5,1-1}$, in (49) has the similar form as $\int_{0}^{\infty} e^{-p x} \ln (a+b x) d x$, replacing the parameters $a, b$, and $p$ with $\bar{\gamma}_{R R}+1,1$, and $1 / \bar{\gamma}_{S 2 R}$, respectively, the integral, $I_{5,1-1}$, can be written as

$$
I_{5,1-1}=\ln \left(\bar{\gamma}_{R R}+1\right)+e^{\frac{\bar{\gamma}_{R R}+1}{\bar{\gamma}_{S 2 R}}} \mathrm{E}_{1}\left(\frac{\bar{\gamma}_{R R}+1}{\bar{\gamma}_{S 2 R}}\right),
$$

$$
\bar{R}_{I R C}^{A F}= \begin{cases}\frac{1}{\ln 2}\left[\left(1+\frac{\bar{\gamma}_{R R}+1}{\bar{\gamma}_{S 1 R}}\right) e^{\frac{\bar{\gamma}_{R R}+1}{\bar{\gamma}_{S 1 R}}} \mathrm{E}_{1}\left(\frac{\bar{\gamma}_{R R}+1}{\bar{\gamma}_{S 1 R}}\right)-1\right], & \frac{\bar{\gamma}_{S 1 R}}{\bar{\gamma}_{R R}+1}=\frac{\bar{\gamma}_{R D 2}}{\bar{\gamma}_{S 2 D 2}+1} \\ \frac{\bar{\gamma}_{S 1 R} e^{\frac{\bar{\gamma}_{S 2 D 2}+1}{\bar{\gamma}_{R D 2}}} \mathrm{E}_{1}\left(\frac{\bar{\gamma}_{S 2 D 2}+1}{\bar{\gamma}_{R D 2}}\right)-\frac{\bar{\gamma}_{R D 2}}{\bar{\gamma}_{S 2 D 2}+1} e^{\frac{\bar{\gamma}_{R R}+1}{\bar{\gamma}_{S 1 R}}} \mathrm{E}_{1}\left(\frac{\bar{\gamma}_{R R}+1}{\bar{\gamma}_{S 1 R}}\right)}{\ln 2\left(\frac{\bar{\gamma}_{S 1 R}}{\bar{\gamma}_{R R}+1}-\frac{\bar{\gamma}_{R D 2}}{\bar{\gamma}_{S 2 D 2}+1}\right)}, & \frac{\bar{\gamma}_{S 1 R}}{\bar{\gamma}_{R R}+1} \neq \frac{\bar{\gamma}_{R D 2}}{\bar{\gamma}_{S 2 D 2}+1} .\end{cases}
$$


and through integration by parts for $x, I_{5,1-2}$ can be rewritten as

$$
\begin{aligned}
I_{5,1-2}= & \underbrace{\int_{0}^{\infty} \ln (z+1) \frac{e^{-z / \bar{\gamma}_{S 1 D 1}}}{\bar{\gamma}_{S 1 D 1}} d z}_{I_{5,1-2-1}} \\
& +\underbrace{\int_{0}^{\infty} \int_{0}^{\infty} \frac{1}{x+z+1} e^{-x / \bar{\gamma}_{R D 1}} \frac{e^{-z / \bar{\gamma}_{S 1 D 1}}}{\bar{\gamma}_{S 1 D 1}} d x d z}_{I_{5,1-2-2}} .
\end{aligned}
$$

Following similar procedure, according to [43, eq. (2.6.23.5)], $I_{5,1-2-1}$ can be expressed as

$$
I_{5,1-2-1}=e^{\frac{1}{\bar{\gamma}_{S 1 D 1}}} \mathrm{E}_{1}\left(\frac{1}{\bar{\gamma}_{S 1 D 1}}\right),
$$

while the integrals $I_{5,1-2-2}$ can be derived as follows.

Special case: (1) when $\bar{\gamma}_{R D 1} \neq \bar{\gamma}_{S 1 D 1}$, the integral, $I_{5,1-2-2}$, can be written as

$$
\begin{aligned}
& I_{5,1-2-2} \stackrel{t=\frac{x+z+1}{z+1}}{=} \frac{1}{\bar{\gamma}_{S 1 D 1}} \int_{0}^{\infty} \int_{1}^{\infty} \frac{1}{t} e^{-\frac{(z+1)(t-1)}{\bar{\gamma}_{R D 1}}} e^{-\frac{z}{\bar{\gamma}_{S 1 D 1}}} d t d \\
& =\frac{1}{\bar{\gamma}_{S 1 D 1}} e^{\frac{1}{\bar{\gamma}_{R D 1}}} \int_{0}^{\infty} e^{-\left(\frac{1}{\bar{\gamma}_{S 1 D 1}}-\frac{1}{\bar{\gamma}_{R D 1}}\right) z} \mathrm{E}_{1}\left(\frac{z+1}{\bar{\gamma}_{R D 1}}\right) d z
\end{aligned}
$$

After solving the integral, $I_{5,1-2-2}$ can be expressed as

$$
\begin{aligned}
I_{5,1-2-2}= & \frac{\bar{\gamma}_{R D 1}}{\left(\bar{\gamma}_{R D 1}-\bar{\gamma}_{S 1 D 1}\right)}\left(e^{\frac{1}{\bar{\gamma}_{R D 1}}} \mathrm{E}_{1}\left(\frac{1}{\bar{\gamma}_{R D 1}}\right)\right. \\
& \left.-e^{\frac{1}{\bar{\gamma}_{S 1 D 1}}} \mathrm{E}_{1}\left(\frac{1}{\bar{\gamma}_{S 1 D 1}}\right)\right) .
\end{aligned}
$$

(2) when $\bar{\gamma}_{R D 1}=\bar{\gamma}_{S 1 D 1}$, the integral, $I_{5,1-2-2}$, can be written as

$$
I_{5,1-2-2}=\frac{1}{\bar{\gamma}_{S 1 D 1}} e^{\frac{1}{\bar{\gamma}_{S 1 D 1}}} \int_{0}^{\infty} \mathrm{E}_{1}\left(\frac{x+1}{\bar{\gamma}_{S 1 D 1}}\right) d x .
$$

[44, eq. (4.1.9)] gives the result of integral form $\int_{0}^{\infty} x^{n} \mathrm{E}_{1}(a x+b) d x$, where $x$ is variable of integration; $n$ is integer; and $a$ and $b$ are constants. Since (55) has the similar form as $\int_{0}^{\infty} x^{n} \mathrm{E}_{1}(a x+b)$, letting $n=0$ and replacing $a$ and $b$ with $\frac{1}{\bar{\gamma}_{S 1 D 1}}$ and $\frac{1}{\bar{\gamma}_{S 1 D 1}}$, respectively, the integral, $I_{5,1-2-2}$, can be expressed as

$$
I_{5,1-2-2}=\frac{1}{\bar{\gamma}_{S 1 D 1}}\left(\bar{\gamma}_{S 1 D 1}-e^{\frac{1}{\bar{\gamma}_{S 1 D 1}}} \mathrm{E}_{1}\left(\frac{1}{\bar{\gamma}_{S 1 D 1}}\right)\right) .
$$

Next, we solve the triple integral $I_{5,2}$. First, through integration by parts for $x$, it can be rewritten as in (57), shown at the bottom of the next page. According to [43, eq. (2.6.23.5)], $I_{5,2-1}$ in (57) can be written as

$$
\begin{aligned}
I_{5,2-1}= & \ln \left(\bar{\gamma}_{R R}+1\right)+e^{\frac{\bar{\gamma}_{R R}+1}{\bar{\gamma}_{S 2 R}}} \mathrm{E}_{1}\left(\frac{\bar{\gamma}_{R R}+1}{\bar{\gamma}_{S 2 R}}\right) \\
& +e^{\frac{1}{\bar{\gamma}_{S 1 D 1}}} \mathrm{E}_{1}\left(\frac{1}{\bar{\gamma}_{S 1 D 1}}\right)
\end{aligned}
$$

and the integral $I_{5,2-2}$ can be solved as follows.

Note, that in order to obtain the bound for the average endto-end rate, we apply Jensen's inequality during deriving the integrals. We first discuss the convexity of integral function, $f(z)=\frac{\left(\bar{\gamma}_{R R}+1\right)}{x\left(\bar{\gamma}_{R R}+1\right)+(z+1)\left(y+\bar{\gamma}_{R R}+1\right)} e^{-\frac{x}{\bar{\gamma}_{R D 1}}} \frac{1}{\bar{\gamma}_{S 2 R}} e^{-\frac{y}{\bar{\gamma}_{S 2 R}}}$. It can be further transformed into such form, $f(z)=a(b z+c)^{-1}$, where $a, b$, and $c$ are constants. Its second derivative is, $f^{\prime \prime}(z)=2 a b^{2}(b z+c)^{-2}>0$. Therefore, integral function $z f(z)$ is convex. We apply Jensen's inequality to eliminate the $\mathrm{z}$-dimension, and perform variable substitution, $t=$ $\frac{x\left(\bar{\gamma}_{R, L I}+1\right)+\left(\bar{\gamma}_{S 1 D 1}+1\right)\left(y+\bar{\gamma}_{R, L I}+1\right)}{\left(\bar{\gamma}_{S 1 D 1}+1\right)\left(y+\bar{\gamma}_{R, L I}+1\right)}$, thus the integral, $I_{5,2-2}$, can be rewritten as

$$
\begin{aligned}
& I_{5,2-2} \\
& \geq \int_{0}^{\infty} \int_{1}^{\infty} e^{\frac{\left(\overline{\left.\gamma_{S 1 D 1}+1\right)\left(y+\bar{\gamma}_{R R}+1\right)}\right.}{\bar{\gamma}_{R D 1}\left(\bar{\gamma}_{R R}+1\right)}} \frac{1}{t} e^{-\frac{t\left(\bar{\gamma}_{S 1 D 1}+1\right)\left(y+\bar{\gamma}_{R R}+1\right)}{\bar{\gamma}_{R D 1}\left(\bar{\gamma}_{R R}+1\right)}} \\
& \quad \times \frac{1}{\bar{\gamma}_{S 2 R}} e^{-\frac{y}{\bar{\gamma}_{S 2 R}}} d t d y \\
& =\int_{0}^{\infty} e^{\frac{\left(\overline{\left.\gamma_{S 1 D 1}+1\right)\left(y+\bar{\gamma}_{R R}+1\right)}\right.}{\bar{\gamma}_{R D 1}\left(\bar{\gamma}_{R R}+1\right)}} \mathrm{E}_{1}\left(\frac{\bar{\gamma}_{S 1 D 1}+1}{\bar{\gamma}_{R D 1}\left(\bar{\gamma}_{R R}+1\right)} y\right. \\
& \left.\quad+\frac{\bar{\gamma}_{S 1 D 1}+1}{\bar{\gamma}_{R D 1}}\right) \frac{1}{\bar{\gamma}_{S 2 R}} e^{-\frac{y}{\bar{\gamma}_{S 2 R}}} d y .
\end{aligned}
$$

We further discuss the integral in the following two cases:

Special case: (1) when $\bar{\gamma}_{R D 1}\left(\bar{\gamma}_{R R}+1\right) \neq \bar{\gamma}_{S 2 R}\left(\bar{\gamma}_{S 1 D 1}+1\right)$, the integral, $I_{5,2-2}$, can be written as

$$
\begin{aligned}
I_{5,2-2} \geq & \frac{1}{\bar{\gamma}_{S 2 R}} e^{\frac{\bar{\gamma}_{S 1 D 1}+1}{\bar{\gamma}_{R D 1}}} \int_{0}^{\infty} e^{-\left(\frac{1}{\bar{\gamma}_{S 2 R}}-\frac{\bar{\gamma}_{S 1 D 1}+1}{\left.\bar{\gamma}_{R D 1} \bar{\gamma}_{R R}+1\right)}\right) y} \\
& \times \mathrm{E}_{1}\left(\frac{\bar{\gamma}_{S 1 D 1}+1}{\bar{\gamma}_{R D 1}\left(\bar{\gamma}_{R R}+1\right)} y+\frac{\bar{\gamma}_{S 1 D 1}+1}{\bar{\gamma}_{R D 1}}\right) d y .
\end{aligned}
$$

$$
\begin{aligned}
\bar{R}_{Y R C, D 1}^{A F}= & \varepsilon\left\{\log _{2}\left(1+\gamma_{Y R C, D 1}^{A F}\right)\right\} \\
= & \int_{0}^{\infty} \int_{0}^{\infty} \int_{0}^{\infty} \log _{2}\left(1+\frac{x y}{x\left(\bar{\gamma}_{R R}+1\right)+(z+1)\left(y+\bar{\gamma}_{R R}+1\right)}\right) g(x, y, z) d x d y d z \\
= & \frac{1}{\ln 2}\{\underbrace{\int_{0}^{\infty} \int_{0}^{\infty} \int_{0}^{\infty} \ln \left(x y+x\left(\bar{\gamma}_{R R}+1\right)+(z+1)\left(y+\bar{\gamma}_{R R}+1\right)\right) g(x, y, z) d x d y d z}_{I_{5,1}} \\
& -\underbrace{\int_{0}^{\infty} \int_{0}^{\infty} \int_{0}^{\infty} \ln \left(x\left(\bar{\gamma}_{R R}+1\right)+(z+1)\left(y+\bar{\gamma}_{R R}+1\right)\right) g(x, y, z) d x d y d z}_{I_{5,2}}\} .
\end{aligned}
$$


After solving the integral, $I_{5,2-2}$ can be further expressed as

$I_{5,2-2} \geq \frac{\bar{\gamma}_{R D 1}\left(\bar{\gamma}_{R R}+1\right)}{\bar{\gamma}_{R D 1}\left(\bar{\gamma}_{R R}+1\right)-\bar{\gamma}_{S 2 R}\left(\bar{\gamma}_{S 1 D 1}+1\right)}$
$\times\left\{e^{\frac{\bar{\gamma}_{S 1 D 1}+1}{\bar{\gamma}_{R D 1}}} \mathrm{E}_{1}\left(\frac{\bar{\gamma}_{S 1 D 1}+1}{\bar{\gamma}_{R D 1}}\right)-e^{\frac{\bar{\gamma}_{R R}+1}{\bar{\gamma}_{S 2 R}}} \mathrm{E}_{1}\left(\frac{\bar{\gamma}_{R R}+1}{\bar{\gamma}_{S 2 R}}\right)\right\}$.

(2) when $\bar{\gamma}_{R D 1}\left(\bar{\gamma}_{R R}+1\right)=\bar{\gamma}_{S 2 R}\left(\bar{\gamma}_{S 1 D 1}+1\right)$, the integral, $I_{5,2-2}$, can be written as

$$
\begin{aligned}
I_{5,2-2} \geq & \frac{1}{\bar{\gamma}_{S 2 R}} e^{\frac{\bar{\gamma}_{S 1 D 1}+1}{\bar{\gamma}_{R D 1}}} \\
& \times \int_{0}^{\infty} \mathrm{E}_{1}\left(\frac{\bar{\gamma}_{S 1 D 1}+1}{\bar{\gamma}_{R D 1}\left(\bar{\gamma}_{R R}+1\right)} y+\frac{\bar{\gamma}_{S 1 D 1}+1}{\bar{\gamma}_{R D 1}}\right) d y .
\end{aligned}
$$

This integral can be evaluated similar to (56), leading to

$$
\begin{aligned}
I_{5,2-2} \geq & \frac{\bar{\gamma}_{R R}+1}{\bar{\gamma}_{S 2 R}\left(\bar{\gamma}_{S 1 D 1}+1\right)}\left\{\bar{\gamma}_{R D 1}\right. \\
& \left.\quad-\left(\bar{\gamma}_{S 1 D 1}+1\right) e^{\frac{\bar{\gamma}_{S 1 D 1}+1}{\bar{\gamma}_{R D 1}}} \mathrm{E}_{1}\left(\frac{\bar{\gamma}_{S 1 D 1}+1}{\bar{\gamma}_{R D 1}}\right)\right\} .
\end{aligned}
$$

Finally, plugging (50), (52), (54), (56), (58), (61) and (63) into (48), $\bar{R}_{Y R C, D 1}^{A F}$ can be written as in (35) and the proof is completed.

\section{APPENDIX F \\ PROOF OF THEOREM 6}

For the $S 1 \rightarrow R \rightarrow D 2$ link, the outage probability of the AF based two-timeslot two-way FD $Y$-relaying can be expressed as in (18). For the $S 2 \rightarrow R \rightarrow D 1$ link, the integral domains for its outage probability consist of $D_{1}=\{(x, y, z) \mid 0<$ $\left.x<\infty, 0<y<\gamma_{t h}\left(\bar{\gamma}_{R R}+1\right), 0<z<\infty\right\}$ and $D_{2}=$ $\left\{(x, y, z) \mid 0<x<\frac{\gamma_{t h}(z+1)\left(y+\bar{\gamma}_{R R}+1\right)}{y-\gamma_{t h}\left(\bar{\gamma}_{R R}+1\right)}, \gamma_{t h}\left(\bar{\gamma}_{R R}+1\right)<\right.$ $y<\infty, 0<z<\infty\}$. Thus, the outage probability of the AF based two-timeslot two-way FD $Y$-relaying can be written as in (64), shown at the bottom of the next page.

Note, that applying Jensen's inequality during deriving the integral, the bound for the outage probability can be obtained. We first discuss the convexity of integral function, $f(z)=\frac{1}{\bar{\gamma}_{S 2 R}} e^{-\frac{y}{\bar{\gamma}_{S 2 R}}} e^{-\frac{\gamma_{t h}(z+1)\left(y+\bar{\gamma}_{R R}+1\right)}{\bar{\gamma}_{R D 1}\left(y-\gamma_{t h}\left(\bar{\gamma}_{R R}+1\right)\right)}}$. It can be transformed into such form, $f(z)=a e^{-(b z+c)}$, where $a, b$, and $c$ are constants, and its second derivative is, $f^{\prime \prime}(z)=$ $a b^{2} e^{-(b z+c)}>0$. Thereby, integral function, $f(z)$, is convex. According to Jensen's inequality and variable substitution, $t=y-\gamma_{t h}\left(\bar{\gamma}_{R R}+1\right)$, the outage probability can be upper bounded as

$$
\begin{aligned}
P_{\text {out }, D 1}^{Y R C, A F}\left(\gamma_{Y R C, D 1}^{A F}<\gamma_{t h}\right) & \\
\leq & 1-\int_{\gamma_{t h}\left(\bar{\gamma}_{R R}+1\right)}^{\infty} \frac{1}{\bar{\gamma}_{S 2 R}} e^{-\frac{y}{\bar{\gamma}_{S 2 R}}} e^{-\frac{\gamma_{t h}\left(\bar{\gamma}_{S 1 D 1}+1\right)\left(y+\bar{\gamma}_{R R}+1\right)}{\bar{\gamma}_{R D 1}\left(y-\gamma_{t h}\left(\bar{\gamma}_{R R}+1\right)\right)}} d y \\
= & 1-\int_{0}^{\infty} \frac{1}{\bar{\gamma}_{S 2 R}} e^{-\left(\frac{t}{\bar{\gamma}_{S 2 R}}+\frac{\gamma_{t h}\left(\gamma_{t h}+1\right)\left(\bar{\gamma}_{S 1 D 1}+1\right)\left(\bar{\gamma}_{R R}+1\right)}{\bar{\gamma}_{R D 1} t}\right)} \\
& \times e^{-\frac{\gamma_{t h}\left(\bar{\gamma}_{R D 1}\left(\bar{\gamma}_{R R}+1\right)+\bar{\gamma}_{S 2 R}(z+1)\right)}{\bar{\gamma}_{S 2 R} \bar{\gamma}_{R D 1}}} d y .
\end{aligned}
$$

After solving the integral, the outage probability can be written as in (37) and the proof is completed.

\section{APPENDIX G \\ PROOF OF THEOREM 7}

For the $S 1 \rightarrow R \rightarrow D 2$ link, the average end-to-end rate for the DF based two-timeslot two-way FD $Y$-relaying over Rayleigh fading channels, can be expressed as in (28). For the $S 2 \rightarrow R \rightarrow D 1$ link, the average rate, $\bar{R}_{Y R C, D 1}^{D F}$, has the similar form as in (27), thus, replacing $\gamma_{S 2 \rightarrow R}$ and $\gamma_{I R C, D 1}^{D F}$ in (27) by (24) and (39), respectively, the average rate can be written as

$\bar{R}_{Y R C, D 1}^{D F}=\varepsilon\left\{\log _{2}\left(1+\min \left(\frac{\gamma_{S 2 R}}{\bar{\gamma}_{R R}+1}, \frac{\gamma_{R D 1}}{\gamma_{S 1 D 1}+1}\right)\right)\right\}$

The integral function, $f\left(\gamma_{S 1 D 1}\right)$ = $\log _{2}\left(1+\min \left(\frac{\gamma_{S 2 R}}{\bar{\gamma}_{R R}+1}, \frac{\gamma_{R D 1}}{\gamma_{S 1 D 1}+1}\right)\right)$, is convex. Applying Jensen's inequality, the average rate can be rewritten as

$\bar{R}_{Y R C, D 1}^{D F} \geq \varepsilon\left\{\log _{2}\left(1+\min \left(\frac{\gamma_{S 2 R}}{\bar{\gamma}_{R R}+1}, \frac{\gamma_{R D 1}}{\bar{\gamma}_{S 1 D 1}+1}\right)\right)\right\}$.

Similar to Theorem 3 and its proof, $\bar{R}_{Y R C, D 1}^{D F}$ can be finally expressed as in (40) and the proof is completed.

\section{APPENDIX H \\ PROOF OF THEOREM 8}

For the $S 1 \rightarrow R \rightarrow D 2$ link, the outage probability of the DF based two-timeslot two-way FD $Y$-relaying is expressed as in (32). For the $S 2 \rightarrow R \rightarrow D 1$ link, similar to (31), the outage probability of that can be written as

$$
\begin{aligned}
P_{\text {out }, D 1}^{Y R C, D F}= & 1-\left(1-\operatorname{Pr}\left(\gamma_{S 2 \rightarrow R}<\gamma_{t h}\right)\right) \\
& \times\left(1-\operatorname{Pr}\left(\gamma_{Y R C, D 1}^{D F}<\gamma_{t h}\right)\right),
\end{aligned}
$$

$$
\begin{aligned}
I_{5,2}= & \underbrace{\int_{0}^{\infty} \int_{0}^{\infty}\left(\ln (z+1)+\ln \left(y+\bar{\gamma}_{R R}+1\right)\right) \frac{e^{-y / \bar{\gamma}_{S 2 R}}}{\bar{\gamma}_{S 2 R}} \frac{e^{-z / \bar{\gamma}_{S 1 D 1}}}{\bar{\gamma}_{S 1 D 1}} d y d z}_{I_{5,2-1}} \\
& +\underbrace{\int_{0}^{\infty} \int_{0}^{\infty} \int_{0}^{\infty} \frac{\left(\bar{\gamma}_{R R}+1\right)}{x\left(\bar{\gamma}_{R R}+1\right)+(z+1)\left(y+\bar{\gamma}_{R R}+1\right)} e^{-x / \bar{\gamma}_{R D 1}} \frac{e^{-y / \bar{\gamma}_{S 2 R}}}{\bar{\gamma}_{S 2 R}} \frac{e^{-z / \bar{\gamma}_{S 1 D 1}}}{\bar{\gamma}_{S 1 D 1}} d x d y d z}_{I_{5,2-2}}
\end{aligned}
$$


where

$$
\begin{aligned}
\operatorname{Pr}\left(\gamma_{S 2 \rightarrow R}<\gamma_{t h}\right) & =\operatorname{Pr}\left(\frac{\gamma_{S 2 R}}{\bar{\gamma}_{R R}+1}<\gamma_{t h}\right) \\
& =\int_{0}^{\gamma_{t h}\left(\bar{\gamma}_{R R}+1\right)} \frac{1}{\bar{\gamma}_{S 2 R}} e^{-\frac{y}{\bar{\gamma}_{S 2 R}} d y} \\
& =1-e^{-\frac{\gamma_{t h}\left(\bar{\gamma}_{R R}+1\right)}{\bar{\gamma}_{S 2 R}}},
\end{aligned}
$$

and according to (39) and [23, eq. (2) and eq. (6)], $\operatorname{Pr}\left(\gamma_{Y R C, D 1}^{D F}<\gamma_{t h}\right)$ can be written as

$$
\operatorname{Pr}\left(\gamma_{Y R C, D 1}^{D F}<\gamma_{t h}\right)=1-\frac{\bar{\gamma}_{R D 1}}{\gamma_{t h} \bar{\gamma}_{S 1 D 1}+\bar{\gamma}_{R D 1}} e^{-\frac{\gamma_{t h}}{\bar{\gamma}_{R D 1}}}
$$

By substituting (69) and (70) into (68), $P_{\text {out }, D 1}^{Y R C, D F}$ can be written as in (41) and the proof is completed.

\section{REFERENCES}

[1] C. Hoymann, W. Chen, J. Montojo, A. Golitschek, C. Koutsimanis, and X. Shen, "Relaying operation in 3GPP LTE: Challenges and solutions," IEEE Commun. Mag., vol. 50, no. 2, pp. 156-162, 2012.

[2] J. Gan et al., "LTE in-band relay prototype and field measurement," in Proc. IEEE VTC (Fall), Yokohama, 2012, pp. 1-5.

[3] Q. Yu et al., "Improving outdoor to indoor coverage by use of TD-LTE in-band relay," in Proc. IEEE PIMRC, London, 2013, pp. 2658-2662.

[4] C. Wang et al., "Cellular architecture and key technologies for 5G wireless communication networks," IEEE Commun. Mag., vol. 52, no. 2, pp. 122130, Feb. 2014.

[5] A. Osseiran et al., "Scenarios for 5G mobile and wireless communications: The vision of the METIS project," IEEE Commun. Mag., vol. 52, no. 5, pp. 26-35, May 2014.

[6] J.-1. Choiy, M. Jainy, K. Srinivasany, P. Levis, and S. Katti, "Achieving single channel, full duplex wireless communication," in Proc. ACM MobiCom, Chicago, 2010, pp. 1-12.

[7] M. Duarte, C. Dick, and A. Sabharwal, "Experiment-driven characterization of full-duplex wireless systems," IEEE Trans. Wireless Commun., vol. 11, no. 12, pp. 4296-4307, Dec. 2012.

[8] S. Hong et al., "Applications of self-interference cancellation in 5G and beyond," IEEE Commun. Mag., vol. 52, no. 2, pp. 114-121, Feb. 2014.

[9] Z. Zhang, X. Chai, K. Long, A. V. Vasilakos, and L. Hanzo, "Full duplex techniques for $5 \mathrm{G}$ networks: Self-interference cancellation, protocol design, and relay selection," IEEE Commun. Mag., vol. 53, no. 5, pp. 128-137, May 2015.

[10] N. J. Laneman, D. N. Tse, and G. W. Wornell, "Cooperative diversity in wireless networks: Efficient protocols and outage behavior," IEEE Trans. Inf. Theory, vol. 50, no. 12, pp. 3062-3080, Dec. 2004.

[11] B. Xia, Y. Fan, J. Thompson, and H. V. Poor, "Buffering in a threenode relay network," IEEE Trans. Wireless Commun., vol. 7, no. 11, pp. 4492-4496, Nov. 2008.

[12] C. Hausl and J. Hagenauer, "Iterative network and channel decoding for the two-way relay channel," in Proc. IEEE ICC, Istanbul, 2006, pp. $1568-1573$.

[13] P. Liu and I.-M. Kim, "Performance analysis of bidirectional communication protocols based on decode-and-forward relaying," IEEE Trans. Commun., vol. 58, no. 9, pp. 2683-2696, Sept. 2010.
[14] R. Ahlswede, N. Cai, S.-Y. R. Li, and R. W. Yeung, "Network information flow," IEEE Trans. Inf. Theory, vol. 46, no. 4, pp. 1204-1216, Jul. 2000.

[15] M. P. Wilson, K. Narayanan, H. D. Pfister, and A. Sprintson, "Joint physical layer coding and network coding for bidirectional relaying," IEEE Trans. Inf. Theory, vol. 56, no. 11, pp. 5641-5654, Nov. 2010.

[16] B. Rankov and A. Wittneben, "Spectral efficient protocols for halfduplex fading relay channels," IEEE J. Sel. Areas Commun., vol. 25 , no. 2, pp. 379-289, Feb. 2007.

[17] T. J. Oechtering, C. Schnurr, I. Bjelakovic, and H. Boche, "Broadcast capacity region of two-phase bidirectional relaying," IEEE Trans. Inf. Theory, vol. 54, no. 1, pp. 454-458, Jan. 2008.

[18] W. Nam, S.-Y. Chung, and Y. H. Lee, "Capacity of the Gaussian twoway relay channel to within $\frac{1}{2}$ bit," IEEE Trans. Inf. Theory, vol. 56, no. 11, pp. 5488-5494, Nov. 2010.

[19] S. S. Ikki and S. Aissa, "Performance analysis of two-way amplifyand-forward relaying in the presence of co-channel interferences," IEEE Trans. Commun., vol. 60, no. 4, pp. 933-939, Apr. 2012.

[20] T. Riihonen, S. Werner, and R. Wichman, "Comparison of full-duplex and half-duplex modes with a fixed amplify-and-forward relay," in Proc. IEEE WCNC, Budapest, 2009, pp. 1-5.

[21] T. Riihonen, S. Werner, and R. Wichman, "Optimized gain control for single-frequency relaying with loop interference," IEEE Trans. Wireless Commun., vol. 8, no. 6, pp. 2801-2806, 2009.

[22] T. Riihonen, S. Werner, and R. Wichman, "Rate-interference trade-off between duplex modes in decode-and-forward relaying," in Proc. IEEE PIMRC, Instanbul, 2010, pp. 690-695.

[23] T. Kwon, S. Lim, S. Choi, and D. Hong, "Optimal duplex mode for DF relay in terms of the outage probability," IEEE Trans. Veh. Technol., vol. 59, no. 7, pp. 3628-3634, Sept. 2010.

[24] T. Riihonen, S. Werner, and R. Wichman, "Hybrid full-duplex/halfduplex relaying with transmit power adaptation," IEEE Trans. Wireless Commun., vol. 10, no. 9, pp. 3074-3085, Sept. 2011.

[25] T. Riihonen, K. Haneda, S. Werner, and R. Wichman, "SINR analysis of full-duplex OFDM repeaters," in Proc. IEEE PIMRC, Tokyo, 2009, pp. 3169-3173.

[26] T. Riihonen, P. Mathecken, and R. Wichman, "Effect of oscillator phase noise and processing delay in full-duplex OFDM repeaters," in Proc. ASILOMAR, Pacific Grove, 2012, pp. 1947-1951.

[27] H. Ju, E. Oh, and D. Hong, "Catching resource-devouring worms in next-generation wireless relay systems: Two-way relay and full-duplex relay," IEEE Commun. Mag., vol. 47, no. 9, pp. 58-65, Sept. 2009.

[28] B. Rankov and A. Wittneben, "Achievable rate regions for the two-way relay channel," in Proc. IEEE ISIT, Seattle, 2006, pp. 1668-1672.

[29] X. Cheng, B. Yu, X. Cheng, and L. Yang, "Two-way full-duplex amplifyand-forward relaying," in Proc. IEEE MILCOM, San Diego, 2013, pp. $1-6$.

[30] B. Niu, M. C. Beluri, Z. Lin, and P. Chitrapu, "Relay assisted cooperative OSTBC communication with SNR imbalance and channel estimation errors," in Proc. IEEE VTC (Spring), Barcelona, 2009, pp. 1-5.

[31] K. Lee, N. Lee, and I. Lee, "Achievable degrees of freedom on K-user $Y$ channel," IEEE Trans. Wireless Commun., vol. 11, no. 3, pp. 1210-1219, Mar. 2012.

[32] A. Chaaban, A. Sezgin, and A. S. Avestimehr, "Approximate sum capacity of the $Y$-channel," IEEE Trans. Inf. Theory, vol. 59, no. 9, pp. 5723-5740, Sept. 2013.

[33] A. Chaaban and A. Sezgin, "The approximate capacity region of the gaussian $Y$-channel via the deterministic approach," IEEE Trans. Inf. Theory, vol. 61, no. 2, pp. 939-962, Feb. 2015.

$$
\begin{aligned}
P_{\text {out }, D 1}^{Y R C, A F}\left(\gamma_{Y R C, D 1}^{A F}<\gamma_{t h}\right)= & \operatorname{Pr}\left(\frac{\gamma_{R D 1} \gamma_{S 2 R}}{\gamma_{R D 1}\left(\bar{\gamma}_{R R}+1\right)+\left(\gamma_{S 1 D 1}+1\right)\left(\gamma_{S 2 R}+\bar{\gamma}_{R R}+1\right)}<\gamma_{t h}\right) \\
= & \int_{0}^{\infty}\left\{\int_{0}^{\gamma_{t h}\left(\bar{\gamma}_{R R}+1\right)} \frac{1}{\bar{\gamma}_{S 2 R}} e^{-\frac{y}{\bar{\gamma}_{S 2 R}}} \int_{0}^{\infty} \frac{1}{\bar{\gamma}_{R D 1}} e^{-\frac{x}{\bar{\gamma}_{R D 1}}} d x d y\right. \\
& \left.+\int_{\gamma_{t h}\left(\bar{\gamma}_{R R}+1\right)}^{\infty} \frac{1}{\bar{\gamma}_{S 2 R}} e^{-\frac{y}{\bar{\gamma}_{S 2 R}}} \int_{0}^{\frac{\gamma_{t h}(z+1)\left(y+\bar{\gamma}_{R R}+1\right)}{y-\gamma_{t h}\left(\bar{\gamma}_{R R}+1\right)}} \frac{1}{\bar{\gamma}_{R D 1}} e^{-\frac{x}{\bar{\gamma}_{R D 1}}} d x d y\right\} \frac{1}{\bar{\gamma}_{S 1 D 1}} e^{-\frac{z}{\bar{\gamma}_{S 1 D 1}}} d z \\
= & 1-\int_{0}^{\infty} \int_{\gamma_{t h}\left(\bar{\gamma}_{R R}+1\right)}^{\infty} \frac{1}{\bar{\gamma}_{S 2 R}} e^{-\frac{y}{\bar{\gamma}_{S 2 R}}} e^{-\frac{\gamma_{t h}(z+1)\left(y+\bar{\gamma}_{R R}+1\right)}{\bar{\gamma}_{R D 1}\left(y-\gamma_{t h}\left(\bar{\gamma}_{R R}+1\right)\right)}} \frac{1}{\bar{\gamma}_{S 1 D 1}} e^{-\frac{z}{\bar{\gamma}_{S 1 D 1}}} d y d z .
\end{aligned}
$$


[34] Q. Wang, Y. Dong, X. Xu, and X. Tao, "Outage probability of fullduplex AF relaying with processing delay and residual self-interference," IEEE Commun. Lett., vol. 19, no. 5, pp. 783-786, May 2015.

[35] C. Wang, H. Farhadi, and M. Skoglund, "Achieving the degrees of freedom of wireless multi-user relay networks," IEEE Trans. Commun., vol. 60 , no. 9 , pp. 2612-2622, Sept. 2012.

[36] T. Riihonen, S. Werner, R. Wichman, "Mitigation of loopback selfInterference in full-duplex MIMO relays," IEEE Trans. Signal Process., vol. 59, no. 12, pp. 5983-5993, Dec. 2011.

[37] L. J. Rodriguez, N. H. Tran, and T. Le-Ngoc, "Performance of fullduplex AF relaying in the presence of residual self-interference," IEEE J. Sel. Areas Commun., vol. 32, no. 9, pp. 1752-1764, Sept. 2014.

[38] R. Hu, C. Hu, J. Jiang, X. Xie, and L. Song, "Full-duplex mode in amplify-and-forward relay channels: Outage probability and ergodic capacity," Int. J. Antennas Propag., 2014.

[39] O. Waqar, D. C. McLernon, and M. Ghogho, "Exact evaluation of ergodic capacity for multihop variable-gain relay networks: A unified framework for generalized fading channels," IEEE Trans. Veh. Technol., vol. 59, no. 8, pp. 4181-4187, Oct. 2010.

[40] L. J. Rodriguez, N. H. Tran, and T. Le-Ngoc, "Capacity and power allocation of dual-hop AF relaying over Rayleigh fading channels," in Proc. IEEE VTC (Fall), Quebec City, 2012, pp. 1-5.

[41] M. O. Hasna and M. -S. Alouini, "Performance analysis of two-hop relayed transmissions over Rayleigh fading channels," in Proc. IEEE VTC (Fall), Vancouver, BC, 2002, pp. 1992-1996.

[42] R. Nikjah and N. C. Beaulieu, "Exact closed-form expressions for the outage probability and ergodic capacity of decode-and-forward opportunistic relaying," in Proc. IEEE GLOBECOM, Honolulu, 2009, pp. 1-8.

[43] A. P. Prudnikov, Yu. A. Brychkov, and O. I. Marichev, Integrals and Series: Elementary Functions, Vol. 1. New York, USA: Gordon \& Breach Sci. Publ., 1986.

[44] M. Geller and E. W. Ng, "A table of integrals of the exponential integral," J. Res. Nat. Bur. Stand. B, vol. 73B, no. 3, July-Sept. 1969. 